This is a self-archived version of an original article. This version may differ from the original in pagination and typographic details.

Author(s): Harrach, Bastian; Pohjola, Valter; Salo, Mikko

Title: Monotonicity and local uniqueness for the Helmholtz equation

Year: 2019

Version: Accepted version (Final draft)

Copyright: @ 2019 Mathematical Sciences Publishers

Rights: In Copyright

Rights url: http://rightsstatements.org/page/InC/1.0/?language=en

Please cite the original version:

Harrach, B., Pohjola, V., \& Salo, M. (2019). Monotonicity and local uniqueness for the Helmholtz equation. Analysis and PDE, 12(7), 2019. https://doi.org/10.2140/apde.2019.12.1741 


\section{ANALYSIS \& PDE}

\section{Volume $12 \quad$ No. $7 \quad 2019$}

Bastian HaRrach, VAlter PoHJOL A AND Mikko Salo

\section{MONOTONICITY AND LOCAL UNIQUENESS \\ FOR THE HELMHOLTZ EQUATION}




\title{
MONOTONICITY AND LOCAL UNIQUENESS FOR THE HELMHOLTZ EQUATION
}

\author{
Bastian Harrach, Valter Pohjola and Mikko Salo
}

\begin{abstract}
This work extends monotonicity-based methods in inverse problems to the case of the Helmholtz (or stationary Schrödinger) equation $\left(\Delta+k^{2} q\right) u=0$ in a bounded domain for fixed nonresonance frequency $k>0$ and real-valued scattering coefficient function $q$. We show a monotonicity relation between the scattering coefficient $q$ and the local Neumann-to-Dirichlet operator that holds up to finitely many eigenvalues. Combining this with the method of localized potentials, or Runge approximation, adapted to the case where finitely many constraints are present, we derive a constructive monotonicitybased characterization of scatterers from partial boundary data. We also obtain the local uniqueness result that two coefficient functions $q_{1}$ and $q_{2}$ can be distinguished by partial boundary data if there is a neighborhood of the boundary part where $q_{1} \geq q_{2}$ and $q_{1} \not \equiv q_{2}$.
\end{abstract}

\section{Introduction}

Let $\Omega \subseteq \mathbb{R}^{n}, n \geq 2$, be a bounded Lipschitz domain with unit outer normal $\nu$. For a fixed nonresonance frequency $k>0$, we study the relation between a real-valued scattering coefficient function $q \in L^{\infty}(\Omega)$ in the Helmholtz equation (or time-independent Schrödinger equation)

$$
\left(\Delta+k^{2} q\right) u=0 \quad \text { in } \Omega
$$

and the local (or partial) Neumann-to-Dirichlet (NtD) operator

$$
\Lambda(q): L^{2}(\Sigma) \rightarrow L^{2}(\Sigma),\left.\quad g \mapsto u\right|_{\Sigma},
$$

where $u \in H^{1}(\Omega)$ solves (1) with Neumann data

$$
\left.\partial_{\nu} u\right|_{\partial \Omega}= \begin{cases}g & \text { on } \Sigma \\ 0 & \text { else. }\end{cases}
$$

Here $\Sigma \subseteq \partial \Omega$ is assumed to be an arbitrary nonempty relatively open subset of $\partial \Omega$. Since $k$ is a nonresonance frequency, $\Lambda(q)$ is well-defined and is easily shown to be a self-adjoint compact operator.

We will show that

$$
q_{1} \leq q_{2} \quad \text { implies } \Lambda\left(q_{1}\right) \leq \text { fin } \Lambda\left(q_{2}\right)
$$

MSC2010: primary 35R30; secondary 35J05.

Keywords: inverse coefficient problems, Helmholtz equation, stationary Schrödinger equation, monotonicity, localized potentials. 
where the inequality on the left-hand side is to be understood pointwise almost everywhere, and the right-hand side denotes that $\Lambda\left(q_{2}\right)-\Lambda\left(q_{1}\right)$ possesses only finitely many negative eigenvalues. Based on a slightly stronger quantitative version of this monotonicity relation, and an extension of the technique of localized potentials [Gebauer 2008] to spaces with finite codimension, we deduce the following local uniqueness result for determining $q$ from $\Lambda(q)$.

Theorem 1.1. Let $O \subseteq \bar{\Omega}$ be a connected relatively open set with $O \cap \Sigma \neq \varnothing$ and $q_{1} \leq q_{2}$ on $O$. Then

$$
\Lambda\left(q_{1}\right)=\Lambda\left(q_{2}\right) \quad \text { implies } \quad q_{1}=q_{2} \quad \text { in } O .
$$

Moreover, if $\left.\left.q_{1}\right|_{o} \not \equiv q_{2}\right|_{o}$, then $\Lambda\left(q_{2}\right)-\Lambda\left(q_{1}\right)$ has infinitely many positive eigenvalues.

Theorem 1.1 will be proven in Section 5. Note that this result removes the assumption $q_{1}, q_{2} \in L_{+}^{\infty}(\Omega)$ from the local uniqueness result in [Harrach and Ullrich 2017], and that it implies global uniqueness if $q_{1}-q_{2}$ is piecewise-analytic; see Corollary 5.2. Note also that in dimension $n=2$, Imanuvilov, Uhlmann and Yamamoto [Imanuvilov et al. 2015] have proven global uniqueness with partial boundary data for potentials $q \in W^{1, p}(\Omega), p>2$. Compared to the result in [Imanuvilov et al. 2015], Theorem 1.1 is both less restrictive, as it holds for $L^{\infty}$-potentials and any dimension $n \geq 2$, and more restrictive, as it relies on a local definiteness condition that is not required in [Imanuvilov et al. 2015].

Additionally to Theorem 1.1, we will also derive a constructive monotonicity-based method to detect a scatterer in an otherwise homogeneous domain. Let the scatterer $D \subseteq \Omega$ be an open set such that $\bar{D} \subseteq \Omega$ and the complement $\Omega \backslash \bar{D}$ is connected, and let

$$
\begin{aligned}
q(x)=1 & \text { for } x \in \Omega \backslash D \text { (a.e.), and } \\
1<q_{\min } \leq q(x) \leq q_{\max } & \text { for } x \in D \text { (a.e.), }
\end{aligned}
$$

with constants $q_{\min }, q_{\max }>0$. For an open set $B \subseteq \Omega$, we define the self-adjoint compact operator

$$
T_{B}: L^{2}(\Sigma) \rightarrow L^{2}(\Sigma), \quad \int_{\Sigma} g T_{B} h \mathrm{~d} s:=\int_{B} k^{2} u_{1}^{(g)} u_{1}^{(h)} \mathrm{d} x,
$$

where $u_{1}^{(g)}, u_{1}^{(h)} \in H^{1}(\Omega)$ solve (1) with $q \equiv 1$ and Neumann data $g, h$ respectively.

Theorem 1.2. For all $0<\alpha \leq q_{\min }-1$,

$$
B \subseteq D \quad \text { if and only if } \quad \alpha T_{B} \leq_{\text {fin }} \Lambda(q)-\Lambda(1) .
$$

We will also give a bound on the number of negative eigenvalues in the case $B \subseteq D$, and prove a similar result for scatterers with negative contrast in Section 6.

Let us give some references on related works and comment on the origins and relevance of our result. The inverse problem considered in this work is closely related to the inverse conductivity problem of determining the positive conductivity function $\gamma$ in the equation $\nabla \cdot(\gamma \nabla u)=0$ in a bounded domain in $\mathbb{R}^{n}$ from knowledge of the associated Neumann-to-Dirichlet operator. This is also known as the problem of electrical impedance tomography or the Calderón problem [1980; 2006]. For a short list of seminal contributions for full boundary data let us refer to [Kohn and Vogelius 1984; 1985; Druskin 1998; Sylvester and Uhlmann 1987; Nachman 1996; Astala and Päivärinta 2006; Haberman and Tataru 
2013; Caro and Rogers 2016]. For the uniqueness problem with partial boundary data there are rather precise results if $n=2$ (see [Imanuvilov et al. 2010; 2015] and the survey [Guillarmou and Tzou 2013]), but in dimensions $n \geq 3$ it is an open question whether measurements on an arbitrary open set $\Sigma \subseteq \partial \Omega$ suffice to determine the unknown coefficient. We refer to [Kenig et al. 2007; Isakov 2007; Kenig and Salo 2013; Krupchyk and Uhlmann 2016] and the overview article [Kenig and Salo 2014] for known results, which either impose strong geometric restrictions on the inaccessible part of the boundary or require measurements of Dirichlet and Neumann data on sets that cover a neighborhood of the so-called front face

$$
F\left(x_{0}\right)=\left\{x \in \partial \Omega:\left(x-x_{0}\right) \cdot v(x) \leq 0\right\}
$$

for a point $x_{0}$ outside the closed convex hull of $\Omega$. Also note that partial boundary data determines full boundary data by unique continuation if there exists a connected neighborhood of the full boundary on which the coefficient is known, so that uniqueness also holds in this case; see [Ammari and Uhlmann 2004].

Theorem 1.1, as well as the previous work [Harrach and Ullrich 2017], give uniqueness results where the measurements are made on an arbitrary open set $\Sigma \subseteq \partial \Omega$. Our result shows that a coefficient change in the positive or negative direction in a neighborhood of $\Sigma$ (or an open subset of $\Sigma$ ) always leads to a change in the Neumann-Dirichlet-operator irrespectively of what happens outside this neighborhood, or the geometry or topology of the domain. Note however that our uniqueness result requires that there is a neighborhood of the boundary part on which the coefficient change is of definite sign. Our uniqueness result does not cover coefficient changes that are infinitely oscillating between positive and negative values when approaching the boundary.

Our result is based on combining monotonicity estimates (similar to those originally derived in [Kang et al. 1997; Ikehata 1998]) with localized potentials. Other theoretical uniqueness results have been obtained by this approach in [Arnold and Harrach 2013; Gebauer 2008; Harrach 2009; 2012; Harrach and Seo 2010; Harrach and Ullrich 2017]. Also note that monotonicity relations have been used in various ways in the study of inverse problems; see, e.g., [Kohn and Vogelius 1984; 1985; Isakov 1988; Alessandrini 1990; Ikehata 1999], where uniqueness results are established by methods that involve monotonicity conditions and blow-up arguments.

Monotonicity-based methods for detecting regions (or inclusions) where a coefficient function differs from a known background have been introduced by Tamburrino and Rubinacci [2002] for the inverse conductivity problem. In that paper they propose to simulate boundary measurements for a number of test regions and then use the fact that a monotonicity relation between the simulated and the true measurements will hold, if the test region lies inside the true inclusion. The work [Harrach and Ullrich 2013] used the technique of localized potentials [Gebauer 2008] to prove that this is really an if-and-only-if relation for the case of continuous measurements modeled by the NtD operator. Moreover, [Harrach and Ullrich 2013] also showed that this if-and-only-if relation still holds when the simulated measurements are replaced by linearized approximations so that the monotonicity method can be implemented without solving any forward problems other than that for the known background medium. For a list of recent works on monotonicity-based methods, let us refer to [Harrach et al. 2015; 2019; Harrach and Ullrich 
2015; Harrach and Minh 2016; 2018; Maffucci et al. 2016; Tamburrino et al. 2016; Barth et al. 2017; Garde 2018; Garde and Staboulis 2017; 2019; Harrach and Lin 2017; Su et al. 2017; Ventre et al. 2017; Brander et al. 2018; Griesmaier and Harrach 2018; Zhou et al. 2018; Harrach and Meftahi 2019; Harrach 2019; Harrach and Lin 2019].

Previous monotonicity-based results often considered second-order equations with positive bilinear forms, such as the conductivity equation. So far, this positivity has been the key to proving monotonicity inequalities between the coefficient and the Neumann-to-Dirichlet operator, and previous results fail to hold in general for equations involving a positive frequency $k>0$ (or a negative potential for the Schrödinger equation). In this article, we remove this limitation and introduce methods for more general elliptic models. We will focus on the Helmholtz equation in a bounded domain as a model case, but the ideas might be applicable to inverse boundary value and scattering problems for, e.g., Helmholtz, Maxwell, and elasticity equations. The main technical novelty of this work is that we treat compact perturbations of positive bilinear forms by extending the monotonicity relations to only hold true up to finitely many eigenvalues, and extend the localized potentials arguments to hold on spaces of finite codimension.

It should also be noted that the localized potentials arguments in [Gebauer 2008] stem from the ideas of the factorization method that was originally developed for scattering problems involving far-field measurements of the Helmholtz equation by Kirsch [1998], see also [Kirsch and Grinberg 2008], and then extended to the inverse conductivity problem in [Brühl and Hanke 2000; Brühl 2001]; see also the overview article [Harrach 2013]. For the inverse conductivity problem, the monotonicity method has the advantage over the factorization method that it allows a convergent regularized numerical implementation, see [Harrach and Ullrich 2013, Remark 3.5; Garde and Staboulis 2019], and that it can also be used for the indefinite case where anomalies of larger and smaller conductivity are present. The localized potentials approach in [Gebauer 2008] has recently been extended to show the possibility of localizing and concentrating electromagnetic fields in [Harrach et al. 2018].

The paper is structured as follows. In Section 2 we discuss the well-posedness of the Helmholtz equation outside resonance frequencies, introduce the Neumann-to-Dirichlet-operators, and give a unique continuation result from sets of positive measure. Sections 3 and 4 contain the main theoretical tools for this work. In Section 3, we introduce a Loewner order of compact self-adjoint operators that holds up to finitely many negative eigenvalues, and show that increasing the scattering index monotonically increases the Neumann-to-Dirichlet-operator in the sense of this new order. We also characterize the connection between the finite number of negative eigenvalues that have to be excluded in the Loewner ordering and the Neumann eigenvalues for the Laplacian. Section 4 extends the localized potentials result from [Gebauer 2008] to the Helmholtz equation and shows that the energy terms appearing in the monotonicity relation can be controlled in spaces of finite codimension. We give two independent proofs of this result, one using a functional analytic relation between operator norms and the ranges of their adjoints, and an alternative proof that is based on a Runge approximation argument. Sections 5 and 6 then contain the main results of this work on local uniqueness for the bounded Helmholtz equation and the detection of scatterers by monotonicity comparisons; see Theorem 1.1 and 1.2 above. 
A preliminary version of these results has been published as the extended abstract [Harrach et al. 2017]. The bound on the number of negative eigenvalues in the monotonicity inequalities derived in this work has recently been improved in [Harrach et al. 2019].

\section{The Helmholtz equation in a bounded domain}

We start by summarizing some properties of the Neumann-to-Dirichlet-operators, discuss well-posedness and the role of resonance frequencies, and state a unique continuation result for the Helmholtz equation in a bounded domain.

2A. Neumann-to-Dirichlet operators. Throughout this work, let $\Omega \subseteq \mathbb{R}^{n}, n \geq 2$, denote a bounded domain with Lipschitz boundary and outer unit normal $v$, and let $\Sigma \subseteq \partial \Omega$ be an open subset of $\partial \Omega$. For a frequency $k \geq 0$ and a real-valued scattering coefficient function $q \in L^{\infty}(\Omega)$, we consider the Helmholtz equation with (partial) Neumann boundary data $g \in L^{2}(\Sigma)$, i.e., finding $u \in H^{1}(\Omega)$ with

$$
\left(\Delta+k^{2} q\right) u=0 \quad \text { in } \Omega,\left.\quad \partial_{\nu} u\right|_{\partial \Omega}= \begin{cases}g & \text { on } \Sigma, \\ 0 & \text { else. }\end{cases}
$$

We also denote the solution by $u_{q}^{(g)}$ instead of $u$ if the choice of $g$ and $q$ is not clear from the context.

The Neumann problem (2) is equivalent to the variational formulation of finding $u \in H^{1}(\Omega)$ such that

$$
\int_{\Omega}\left(\nabla u \cdot \nabla v-k^{2} q u v\right) \mathrm{d} x=\left.\int_{\partial \Omega} g v\right|_{\partial \Omega} \mathrm{d} s \quad \text { for all } v \in H^{1}(\Omega) .
$$

We introduce the bounded linear operators

$$
\begin{gathered}
I: H^{1}(\Omega) \rightarrow H^{1}(\Omega), \\
j: H^{1}(\Omega) \rightarrow L^{2}(\Omega), \\
M_{q}: L^{2}(\Omega) \rightarrow L^{2}(\Omega),
\end{gathered}
$$

where $I$ denotes the identity operator, $j$ is the compact embedding from $H^{1}$ to $L^{2}$, and $M_{q}$ is the multiplication operator by $q$. We furthermore use $\langle\cdot, \cdot\rangle$ to denote the $H^{1}(\Omega)$ inner product and define the operators

$$
K:=j^{*} j \quad \text { and } \quad K_{q}:=j^{*} M_{q} j
$$

which are compact self-adjoint linear operators from $H^{1}(\Omega)$ to $H^{1}(\Omega)$. By

$$
\gamma_{\Sigma}: H^{1}(\Omega) \rightarrow L^{2}(\Sigma),\left.\quad v \mapsto v\right|_{\Sigma}
$$

we denote the compact trace operator.

With this notation (3) can be written as

$$
\left\langle\left(I-K-k^{2} K_{q}\right) u, v\right\rangle=\int_{\partial \Omega} g\left(\gamma_{\Sigma} v\right) \mathrm{d} s \quad \text { for all } v \in H^{1}(\Omega),
$$

so that the Neumann problem for the Helmholtz equation (2) is equivalent to the equation

$$
\left(I-K-k^{2} K_{q}\right) u=\gamma_{\Sigma}^{*} g .
$$


Our results on identifying the scattering coefficient $q$ will require that $I-K-k^{2} K_{q}$ is continuously invertible, which is equivalent to the fact that $k$ is not a resonance frequency, or, equivalently; that $0 i s$ not a Neumann eigenvalue, see Lemmas 2.2 and 3.10. Note that this implies, in particular, that $k>0$ and $q \not \equiv 0$. We can then define the Neumann-to-Dirichlet operator (with Neumann data prescribed and Dirichlet data measured on the same open subset $\Sigma \subseteq \partial \Omega$ )

$$
\Lambda(q): L^{2}(\Sigma) \rightarrow L^{2}(\Sigma),\left.\quad g \mapsto u\right|_{\Sigma}, \quad \text { where } u \in H^{1}(\Omega) \text { solves (2). }
$$

The Neumann-to-Dirichlet operator satisfies

$$
\Lambda(q)=\gamma_{\Sigma}\left(I-K-k^{2} K_{q}\right)^{-1} \gamma_{\Sigma}^{*}
$$

which shows that $\Lambda(q)$ is a compact self-adjoint linear operator.

We will show in Section 3 that there is a monotonicity relation between the scattering coefficient $q$ and the Neumann-to-Dirichlet-operator $\Lambda(q)$. Increasing $q$ will increase $\Lambda(q)$ in the sense of operator definiteness up to finitely many eigenvalues. The number of eigenvalues that do not follow the increase will be bounded by the number defined in the following lemma. Note that here, and throughout the paper, we always count the number of eigenvalues of a compact self-adjoint operator with multiplicity according to the dimension of the associated eigenspaces.

Lemma 2.1. Given $k>0$ and $q \in L^{\infty}(\Omega)$, let $d(q)$ be the number of eigenvalues of $K+k^{2} K_{q}$ that are larger than 1 , and let $V(q)$ be the sum of the associated eigenspaces. Then $d(q)=\operatorname{dim}(V(q)) \in \mathbb{N}_{0}$ is finite, and

$$
\int_{\Omega}\left(|\nabla v|^{2}-k^{2} q|v|^{2}\right) \mathrm{d} x \geq 0 \quad \text { for all } v \in V(q)^{\perp},
$$

where $V(q)^{\perp}$ denotes the orthocomplement of $V(q)$ in $H^{1}(\Omega)$.

Proof. Since

$$
\left\langle\left(I-K-k^{2} K_{q}\right) v, v\right\rangle=\int_{\Omega}\left(|\nabla v|^{2}-k^{2} q|v|^{2}\right) \mathrm{d} x,
$$

the assertion follows from the spectral theorem for compact self-adjoint operators.

We will show in Lemma 3.10 that $d(q)$ agrees with the number of positive Neumann eigenvalues of $\Delta+k^{2} q$. If $q(x) \leq q_{\max } \in \mathbb{R}$ for all $x \in \Omega$ (a.e.) then $d(q) \leq d\left(q_{\max }\right)$, and $d\left(q_{\max }\right)$ is the number of Neumann eigenvalues of the Laplacian $\Delta$ that are larger than $-k^{2} q_{\max }$; see Corollary 3.11.

2B. Resonance frequencies. We now summarize some results on the solvability of the Helmholtz equation (2) outside of resonance frequencies.

Lemma 2.2. Let $q \in L^{\infty}(\Omega)$.

(a) For each $k \geq 0$, the following properties are equivalent:

(i) For each $F \in L^{2}(\Omega)$ and $g \in L^{2}(\partial \Omega)$, there exists a unique solution $u \in H^{1}(\Omega)$ of

$$
\left(\Delta+k^{2} q\right) u=F \quad \text { in } \Omega,\left.\quad \partial_{\nu} u\right|_{\partial \Omega}=g,
$$

and the solution depends linearly and continuously on $F$ and $g$. 
(ii) The homogeneous Neumann problem

$$
\left(\Delta+k^{2} q\right) u=0 \quad \text { in } \Omega,\left.\quad \partial_{\nu} u\right|_{\partial \Omega}=0,
$$

admits only the trivial solution $u \equiv 0$.

(iii) The operator $I-K-k^{2} K_{q}: H^{1}(\Omega) \rightarrow H^{1}(\Omega)$ is continuously invertible.

We call $k$ a resonance frequency if the properties (i)-(iii) do not hold.

(b) If $q \not \equiv 0$, then the set of resonance frequencies is countable and discrete.

Proof. (a) Clearly, (i) implies (ii), and, using the equivalence of (2) and (4), (ii) implies that $I-K-k^{2} K_{q}$ is injective. Since $K$ and $K_{q}$ are compact, the operator $I-K-k^{2} K_{q}$ is Fredholm of index 0 . Hence, injectivity of $I-K-k^{2} K_{q}$ already implies that $I-K-k^{2} K_{q}$ is continuously invertible, so that (ii) implies (iii). Finally, $u \in H^{1}(\Omega)$ solves (6) if and only if

$$
\int_{\Omega}\left(\nabla u \cdot \nabla v-k^{2} q u v\right) \mathrm{d} x=-\int_{\Omega} F v \mathrm{~d} x+\left.\int_{\partial \Omega} g v\right|_{\partial \Omega} \mathrm{d} S \quad \text { for all } v \in H^{1}(\Omega) .
$$

This is equivalent to

$$
\left\langle\left(I-K-k^{2} K_{q}\right) u, v\right\rangle=-\int_{\Omega} F j(v) \mathrm{d} x+\int_{\partial \Omega} g \gamma_{\partial \Omega}(v) \mathrm{d} s \quad \text { for all } v \in H^{1}(\Omega),
$$

and thus equivalent to

$$
\left(I-K-k^{2} K_{q}\right) u=-j^{*} F+\gamma_{\partial \Omega}^{*} g
$$

so that (iii) implies (i).

(b) We extend $I, K$, and $K_{q}$ to the Sobolev space of complex-valued functions

$$
I, K, K_{q}: H^{1}(\Omega ; \mathbb{C}) \rightarrow H^{1}(\Omega ; \mathbb{C})
$$

For $k \in \mathbb{C}$ we then define

$$
R(k):=K+k^{2} K_{q}: H^{1}(\Omega ; \mathbb{C}) \rightarrow H^{1}(\Omega ; \mathbb{C})
$$

$R(k)$ is a family of compact operators depending analytically on $k \in \mathbb{C}$. The analytic Fredholm theorem, see, e.g., [Reed and Simon 1972, Theorem VI.14], now implies that either $I-R(k)$ is not invertible for all $k \in \mathbb{C}$, or that there is a countable discrete set $Z \subseteq \mathbb{C}$ such that $I-R(k)$ is continuously invertible when $k \in \mathbb{C} \backslash Z$. Hence, to prove (b), it suffices to show that there exists $k \in \mathbb{C}$ for which $I-R(k)$ is invertible.

We will show that this is the case for any $0 \neq k \in \mathbb{C}$ with $\operatorname{Re}\left(k^{2}\right)=0$. In fact, $(I-R(k)) u=0$ implies

$$
0=\int_{\Omega}\left(\nabla u \cdot \nabla v-k^{2} q u v\right) \mathrm{d} x \quad \text { for all } v \in H^{1}(\Omega ; \mathbb{C}) .
$$

Using $v:=\bar{u}$ and taking the real part yields that $0=\int_{\Omega}|\nabla u|^{2} \mathrm{~d} x$, which shows that $u$ must be constant, and that

$$
\int_{\Omega} k^{2} q u v \mathrm{~d} x=0 \quad \text { for all } v \in H^{1}(\Omega ; \mathbb{C}) .
$$


Together with $k^{2} \neq 0$, and $q \neq \equiv$, this shows that $u \equiv 0$. Hence, $I-R(k)$ is injective and thus invertible for all $0 \neq k \in \mathbb{C}$ with $\operatorname{Re}\left(k^{2}\right)=0$.

2C. Unique continuation. We will make use of a unique continuation property for the Helmholtz equation from sets of positive measure. In two dimensions, this follows from a standard reduction to quasiconformal mappings. However, since we could not find a proof in the literature, we will first give the argument following [Alessandrini 2012] and references therein (in fact [Alessandrini 2012] proves strong unique continuation for more general equations). See also [Astala et al. 2009] for basic facts on quasiconformal mappings in the plane.

Lemma 2.3. Let $\Omega \subset \mathbb{R}^{2}$ be a connected open set, and suppose that $u \in H_{\mathrm{loc}}^{1}(\Omega)$ is a weak solution of

$$
-\operatorname{div}(A \nabla u)+d u=0 \quad \text { in } \Omega,
$$

where $A \in L^{\infty}\left(\Omega, \mathbb{R}^{n \times n}\right)$ is symmetric and satisfies $A(x) \xi \cdot \xi \geq c_{0}|\xi|^{2}$ for some $c_{0}>0$, and $d \in L^{q / 2}(\Omega)$ for some $q>2$. If $u$ vanishes in a set $E$ of positive measure, then $u \equiv 0$ in $\Omega$.

Proof. It is enough to show that $u$ vanishes in some ball, since then weak (or strong) unique continuation [Alessandrini 2012] implies that $u \equiv 0$. Let $x_{0}$ be a point of density 1 in $E$ and let $U_{r}:=B_{r}\left(x_{0}\right)$ and $E_{r}:=E \cap U_{r}$. There is $r_{0}>0$ so that if $r<r_{0}$, then $U_{r} \subset \Omega$ and $E_{r}$ has positive measure.

We will now work in $U_{r}$. Observe first that there is $p>2$ so that $u \in W^{1, p}\left(U_{r}\right)$ [Astala et al. 2009, Theorem 16.1.4]. In particular $u$ is Hölder continuous and we may assume (after removing a set of measure zero from $E$ ) that $u(x)=0$ for all $x \in E_{r}$. The first step is to show that $\nabla u=0$ a.e. on $E_{r}$. Let $N_{1}$ be the set of points in $E_{r}$ where $u$ is not differentiable, and let $N_{2}$ be the set of points of density $<1$ in $E_{r}$. Then $N_{1}$ and $N_{2}$ have zero measure. Fix a point $x \in E_{r} \backslash\left(N_{1} \cup N_{2}\right)$ and a unit direction $e$. There is a sequence $\left(x_{j}\right)$ with $x_{j} \in B(x, 1 / j) \cap E_{r}$ so that $\left|\left(x_{j}-x\right) /\right| x_{j}-x|-e| \leq 1 / j$ for $j$ large (for if not, then all points in $E_{r}$ near $x$ would be outside a fixed sector in direction $e$, which contradicts the fact that $x$ has density 1 ). Since $u$ is differentiable at $x$,

$$
u\left(x_{j}\right)-u(x)=\nabla u(x) \cdot\left(x_{j}-x\right)+o\left(\left|x-x_{j}\right|\right) .
$$

Dividing by $\left|x-x_{j}\right|$ and using that $u\left(x_{j}\right)=u(x)=0$ implies that $\nabla u(x) \cdot e=0$. It follows that $\nabla u$ vanishes in $E_{r} \backslash\left(N_{1} \cup N_{2}\right)$, so indeed

$$
u=0 \quad \text { in } E_{r}, \quad \nabla u=0 \quad \text { a.e. in } E_{r} .
$$

The next step is to reduce to the case where $d=0$. As in [Alessandrini 2012, Proposition 2.4], we choose $r$ small enough so that there is a nonvanishing $w \in W^{1, p}\left(U_{r}\right)$ satisfying

$$
\begin{gathered}
-\operatorname{div}(A \nabla w)+d w=0 \quad \text { in } U_{r}, \\
\frac{1}{2} \leq w \leq 2 \quad \text { in } U_{r}, \quad\|\nabla w\|_{L^{p}\left(U_{r}\right)} \leq 1 .
\end{gathered}
$$

We write $v=u / w$. It follows that $v \in W^{1, p}\left(U_{r}\right)$ is a weak solution of

$$
-\operatorname{div}(\tilde{A} \nabla v)=0 \quad \text { in } U_{r},
$$


where $\tilde{A}=w^{2} A$ is $L^{\infty}$ and uniformly elliptic. Moreover, (8) implies that

$$
v=0 \quad \text { in } E_{r}, \quad \nabla v=0 \quad \text { a.e. in } E_{r} .
$$

To prove the lemma, we will show that $v \equiv 0$ in some ball.

Let $J=\left(\begin{array}{cc}0 & -1 \\ 1 & 0\end{array}\right)$. Since $\tilde{A} \nabla v$ is divergence-free, there is a real-valued function $\tilde{v} \in H^{1}\left(U_{r}\right)$ satisfying

$$
\nabla \tilde{v}=J(\tilde{A} \nabla v) .
$$

Such a function $\tilde{v}$ is unique up to an additive constant. Define

$$
f=v+i \tilde{v} .
$$

As in [Alessandrini 2012], $f \in H^{1}\left(U_{r}\right)$ solves an equation of the form

$$
\partial_{\bar{z}} f=\mu \partial_{z} f+v \bar{\partial}_{z} f \quad \text { in } U_{r},
$$

where $\|\mu\|_{L^{\infty}\left(U_{r}\right)}+\|v\|_{L^{\infty}\left(U_{r}\right)}<1$. It follows that $f$ is a quasiregular map and by the Stoilow factorization [Astala et al. 2009, Theorem 5.5.1] it has the representation

$$
f(z)=F(\chi(z)), \quad z \in U,
$$

where $\chi$ is a quasiconformal map $\mathbb{C} \rightarrow \mathbb{C}$ and $F$ is a holomorphic function on $\chi(U)$.

Finally, the Jacobian determinant $J_{f}$ of $f$ is given by

$$
J_{f}(z)=F^{\prime}(\chi(z)) J_{\chi}(z) .
$$

Using (9) and (10), we see that $J_{f}=0$ a.e. in $E_{r}$. Moreover, since $\chi$ is quasiconformal, $J_{\chi}$ can only vanish in a set of measure zero [Astala et al. 2009, Corollary 3.7.6]. It follows that $F^{\prime}(\chi(z))=0$ for a.e. $z \in E_{r}$. Then the Taylor series of the analytic function $F^{\prime}$ at $\chi\left(x_{0}\right)$ must vanish (otherwise one would have $F^{\prime}(\chi(z))=\left(\chi(z)-\chi\left(x_{0}\right)\right)^{N} g(\chi(z))$, where $g\left(\chi\left(x_{0}\right)\right) \neq 0$ and the only zero near $x_{0}$ would be $\left.z=x_{0}\right)$. Thus $F^{\prime}=0$ near $x_{0}$, so $F$ is constant, $f$ is also constant, and $v=0$ near $x_{0}$.

We can now state the unique continuation property for any dimension $n \geq 2$ in the form that we will utilize in the later sections. As in [Harrach and Ullrich 2013, Definition 2.2] we say that a relatively open subset $O \subseteq \bar{\Omega}$ is connected to $\Sigma$ if $O$ is connected and $\Sigma \cap O \neq \varnothing$.

Theorem 2.4. (a) Let $u \in H^{1}(\Omega)$ solve

$$
\left(\Delta+k^{2} q\right) u=0 \quad \text { in } \Omega .
$$

If $\left.u\right|_{E}=0$ for a subset $E \subseteq \Omega$ with positive measure then $u(x)=0$ for all $x \in \Omega$ (a.e.)

(b) Let $u \in H^{1}(\Omega), \Delta u \in L^{2}(\Omega)$, and

$$
\left(\Delta+k^{2} q\right) u=0 \quad \text { in } \Omega \backslash C
$$

for a closed set $C$ for which $\bar{\Omega} \backslash C$ is connected to $\Sigma$. If $\left.u\right|_{\Sigma}=0$ and $\left.\partial_{\nu} u\right|_{\Sigma}=0$, then $u(x)=0$ for all $x \in \Omega \backslash C$ (a.e.) 
Proof. For $n=2$, (a) follows from Lemma 2.3. For $n \geq 3$, (a) is shown in [Harrach and Ullrich 2017, Theorem 4.2] (see also [Regbaoui 2001, proof of Theorem 2.1]) by combining the following two results:

(i) If $u \in H^{1}(\Omega)$ solves (11) and vanishes on a measurable set of positive measure then $u$ has a zero of infinite order; see, e.g., [de Figueiredo and Gossez 1992, Proposition 3; Hadi and Tsouli 2001, Theorem 2.1].

(ii) The trivial solution $u \equiv 0$ is the only $H^{1}(\Omega)$-solution of (11) that has a zero of infinite order; see, e.g, [Hörmander 1985, Theorem 17.2.6].

Part (b) follows from (a) by extending $u$ by zero on $B \backslash \Omega$, where $B$ is a small ball with $B \cap \partial \Omega \subseteq \Sigma$; see the proof of Lemma 4.4(c) in [Harrach and Ullrich 2017].

\section{Monotonicity and localized potentials for the Helmholtz equation}

In this section we show that increasing the scattering coefficient leads to a larger Neumann-to-Dirichlet operator in a certain sense. For this result, the Neumann-to-Dirichlet operators are ordered by an extension of the Loewner order of compact self-adjoint operators that holds up to finitely many negative eigenvalues.

3A. A Loewner order up to finitely many eigenvalues. We start by giving a rigorous definition and characterization of this ordering.

Definition 3.1. Let $A, B: X \rightarrow X$ be two self-adjoint compact linear operators on a Hilbert space $X$. For a number $d \in \mathbb{N}_{0}$, we write

$$
A \leq_{d} B \quad \text { or } \quad\langle A x, x\rangle \leq_{d}\langle B x, x\rangle
$$

if $B-A$ has at most $d$ negative eigenvalues. We also write $A \leq_{\text {fin }} B$ if $A \leq_{d} B$ holds for some $d \in \mathbb{N}_{0}$, and we write $A \leq B$ if $A \leq_{d} B$ holds for $d=0$.

Note that for $d=0$ this is the standard partial ordering of compact self-adjoint operators in the sense of operator definiteness (also called Loewner order). Also note that " $\leq_{\text {fin }}$ " and " $\leq_{d}$ " (for $d \neq 0$ ) are not partial orders since they are clearly not antisymmetric. Obviously, " $\leq$ fin" and " $\leq d$ " are reflexive, and " $\leq$ fin" is also transitive (see Lemma 3.4 below) and thus a so-called preorder.

To characterize this new ordering, we will make use of the following lemma.

Lemma 3.2. Let $A: X \rightarrow X$ be a self-adjoint compact linear operator on a Hilbert space $X$ with inner product $\langle\cdot, \cdot\rangle$ inducing the norm $\|\cdot\|$. Let $d \in \mathbb{N}_{0}$ and $r \in \mathbb{R}, r \geq 0$.

(a) The following statements are equivalent:

(i) A has at most d eigenvalues larger than $r$.

(ii) There exists a compact self-adjoint operator $F: X \rightarrow X$ with

$$
\operatorname{dim}(\mathcal{R}(F)) \leq d \quad \text { and } \quad\langle(A-F) x, x\rangle \leq r\|x\|^{2} \quad \text { for all } x \in X,
$$

where $\mathcal{R}(F)$ stands for the range of $F$. 
(iii) There exists a subspace $W \subset X$ with $\operatorname{codim}(W) \leq d$ such that

$$
\langle A w, w\rangle \leq r\|w\|^{2} \quad \text { for all } w \in W
$$

(iv) There exists a subspace $V \subset X$ with $\operatorname{dim}(V) \leq d$ such that

$$
\langle A v, v\rangle \leq r\|v\|^{2} \quad \text { for all } v \in V^{\perp} .
$$

(b) The following statements are equivalent:

(i) A has (at least) d eigenvalues larger than $r$.

(ii) There exists a subspace $V \subset X$ with $\operatorname{dim}(V) \geq d$ such that

$$
\langle A v, v\rangle>r\|v\|^{2} \quad \text { for all } v \in V .
$$

Proof. (a) We start by showing that (i) implies (ii). Let $A$ have at most $d$ eigenvalues larger than $r \geq 0$. Let $\left(\lambda_{k}\right)_{k \in \mathbb{N}}$ be the nonzero eigenvalues of $A$, ordered in such a way that $\lambda_{k} \leq r$ for $k>d$. Let $\mathcal{N}(A)$ denote the kernel of $A$ and let $\left(v_{k}\right)_{k \in \mathbb{N}} \in X$ be a sequence of corresponding eigenvectors forming an orthonormal basis of $\mathcal{N}(A)^{\perp}$. Then

$$
A x=\sum_{k=1}^{\infty} \lambda_{k} v_{k}\left\langle v_{k}, x\right\rangle \quad \text { for all } x \in X,
$$

and (ii) follows with $F: X \rightarrow X$ defined by

$$
F: x \mapsto \sum_{k=1}^{d} \lambda_{k} v_{k}\left\langle v_{k}, x\right\rangle \quad \text { for all } x \in X .
$$

The implication from (ii) to (iii) follows by setting $W:=\mathcal{N}(F)$ since

$$
\operatorname{codim}(W)=\operatorname{dim}\left(W^{\perp}\right)=\operatorname{dim}(\mathcal{R}(F)) \leq d
$$

and

$$
\langle A w, w\rangle=\langle(A-F) w, w\rangle \geq 0 .
$$

Part (iii) implies (iv) by setting $V:=W^{\perp}$.

To show that (iv) implies (i), we assume that (i) is not true, so that $A$ has at least $d+1$ eigenvalues larger than $r \geq 0$. We sort the positive eigenvalues of $A$ in decreasing order to obtain

$$
\lambda_{1} \geq \cdots \geq \lambda_{d} \geq \lambda_{d+1}>r .
$$

Then, by the Courant-Fischer-Weyl min-max principle, see, e.g., [Lax 2002, p. 318], we have that the minimum over all $d$-dimensional subspaces $V \subset X$ must satisfy

$$
\min _{\substack{V \subset X \\ \operatorname{dim}(V)=d}} \max _{\substack{v \in V^{\perp} \\\|v\|=1}}\langle A v, v\rangle=\lambda_{d+1}>r,
$$

which shows that (iv) cannot be true. Hence, (iv) implies (i). 
(b) This can be shown analogously to (a). Part (ii) follows from (i) by choosing $V$ as the sum of eigenspaces for eigenvalues larger than $r$, and (ii) implies (i) by using the Courant-Fischer-Weyl min-max principle.

Corollary 3.3. Let $A, B: X \rightarrow X$ be two self-adjoint compact linear operators on a Hilbert space $X$ with inner product $\langle\cdot, \cdot\rangle$. For any number $d \in \mathbb{N}_{0}$, the following statements are equivalent:

(a) $A \leq_{d} B$

(b) There exists a compact self-adjoint operator $F: X \rightarrow X$ with

$$
\operatorname{dim}(\mathcal{R}(F)) \leq d \quad \text { and } \quad\langle(B-A+F) x, x\rangle \geq 0 \quad \text { for all } x \in X .
$$

(c) There exists a subspace $W \subset X$ with $\operatorname{codim}(W) \leq d$ such that

$$
\langle(B-A) w, w\rangle \geq 0 \quad \text { for all } w \in W .
$$

(d) There exists a subspace $V \subset X$ with $\operatorname{dim}(V) \leq d$ such that

$$
\langle(B-A) v, v\rangle \geq 0 \quad \text { for all } v \in V^{\perp} .
$$

Proof. This follows from Lemma 3.2(a) with $r=0$ and $A$ replaced by $A-B$.

Lemma 3.4. Let $A, B, C: X \rightarrow X$ be self-adjoint compact linear operators on a Hilbert space $X$. For $d_{1}, d_{2} \in \mathbb{N}_{0}$

$$
\begin{aligned}
& A \leq d_{1} B \quad \text { and } B \leq_{d_{2}} C \quad \text { implies } A \leq_{d_{1}+d_{2}} C \text {, } \\
& A \leq_{\text {fin }} B \text { and } B \leq_{\text {fin }} C \text { implies } A \leq_{\text {fin }} C \text {. }
\end{aligned}
$$

Proof. This follows from the characterization in Corollary 3.3(b).

3B. A monotonicity relation for the Helmholtz equation. With this new ordering, we can show a monotonicity relation between the scattering index and the Neumann-to-Dirichlet-operators. Note that the dimension bound in the last line of the following theorem has recently been improved to $d\left(q_{2}\right)-d\left(q_{1}\right)$ in [Harrach et al. 2019].

Theorem 3.5. Let $q_{1}, q_{2} \in L^{\infty}(\Omega) \backslash\{0\}$. Assume that $k>0$ is not a resonance for $q_{1}$ or $q_{2}$, and let $d\left(q_{2}\right) \in \mathbb{N}_{0}$ be defined as in Lemma 2.1.

Then there exists a subspace $V \subset L^{2}(\Sigma)$ with $\operatorname{dim}(V) \leq d\left(q_{2}\right)$ such that

$$
\int_{\Sigma} g\left(\Lambda\left(q_{2}\right)-\Lambda\left(q_{1}\right)\right) g \mathrm{~d} s \geq \int_{\Omega} k^{2}\left(q_{2}-q_{1}\right)\left|u_{1}^{(g)}\right|^{2} \mathrm{~d} x \quad \text { for all } g \in V^{\perp} \text {. }
$$

In particular

$$
q_{1} \leq q_{2} \quad \text { implies } \Lambda\left(q_{1}\right) \leq d\left(q_{2}\right) \Lambda\left(q_{2}\right) .
$$

Remark 3.6. Note that by interchanging $q_{1}$ and $q_{2}$, Theorem 3.5 also yields that there exists a subspace $V \subset L^{2}(\Sigma)$ with $\operatorname{dim}(V) \leq d\left(q_{1}\right)$ such that

$$
\int_{\Sigma} g\left(\Lambda\left(q_{2}\right)-\Lambda\left(q_{1}\right)\right) g \mathrm{~d} s \leq \int_{\Omega} k^{2}\left(q_{2}-q_{1}\right)\left|u_{2}^{(g)}\right|^{2} \mathrm{~d} x \quad \text { for all } g \in V^{\perp} .
$$


To prove Theorem 3.5 we will use the following lemmas.

Lemma 3.7. Let $q_{1}, q_{2} \in L^{\infty}(\Omega) \backslash\{0\}$. Assume that $k>0$ is not a resonance for $q_{1}$ or $q_{2}$. Then, for all $g \in L^{2}(\Sigma)$,

$$
\int_{\Sigma} g\left(\Lambda\left(q_{2}\right)-\Lambda\left(q_{1}\right)\right) g \mathrm{~d} s+\int_{\Omega} k^{2}\left(q_{1}-q_{2}\right)\left|u_{1}^{(g)}\right|^{2} \mathrm{~d} x=\int_{\Omega}\left(\left|\nabla\left(u_{2}^{(g)}-u_{1}^{(g)}\right)\right|^{2}-k^{2} q_{2}\left|u_{2}^{(g)}-u_{1}^{(g)}\right|^{2}\right) \mathrm{d} x,
$$

where $u_{1}^{(g)}, u_{2}^{(g)}$ solve the Helmholtz equation (2) with Neumann boundary data $g$ and $q=q_{1}, q=q_{2}$ respectively.

Proof. Define the bilinear form

$$
B_{q}(u, v)=\int_{\Omega}\left(\nabla u \cdot \nabla v-k^{2} q u v\right) \mathrm{d} x, \quad u, v \in H^{1}(\Omega) .
$$

Writing $u_{1}=u_{1}^{(g)}$ and $u_{2}=u_{2}^{(g)}$, from the definition of the NtD map and from (3) we have

$$
\int_{\Sigma} g \Lambda\left(q_{1}\right) g \mathrm{~d} s=\int_{\Sigma}\left(\partial_{\nu} u_{1}\right) u_{1} \mathrm{~d} s=2 \int_{\Sigma}\left(\partial_{\nu} u_{2}\right) u_{1} \mathrm{~d} s-\int_{\Sigma}\left(\partial_{\nu} u_{1}\right) u_{1} \mathrm{~d} s=2 B_{q_{2}}\left(u_{2}, u_{1}\right)-B_{q_{1}}\left(u_{1}, u_{1}\right)
$$

and

$$
\int_{\Sigma} g \Lambda\left(q_{2}\right) g \mathrm{~d} s=\int_{\Sigma}\left(\partial_{\nu} u_{2}\right) u_{2} \mathrm{~d} s=B_{q_{2}}\left(u_{2}, u_{2}\right)
$$

We thus obtain that

$$
\begin{aligned}
\int_{\Sigma} g\left(\Lambda\left(q_{2}\right)-\Lambda\left(q_{1}\right)\right) g \mathrm{~d} s & =B_{q_{2}}\left(u_{2}, u_{2}\right)-2 B_{q_{2}}\left(u_{2}, u_{1}\right)+B_{q_{1}}\left(u_{1}, u_{1}\right) \\
& =B_{q_{2}}\left(u_{2}-u_{1}, u_{2}-u_{1}\right)-B_{q_{2}}\left(u_{1}, u_{1}\right)+B_{q_{1}}\left(u_{1}, u_{1}\right) .
\end{aligned}
$$

We will show that the bilinear forms in the right-hand sides in Lemma 3.7 are positive up to a finite-dimensional subspace.

Lemma 3.8. Let $q_{1}, q_{2} \in L^{\infty}(\Omega) \backslash\{0\}$ for which $k>0$ is not a resonance. There exists a subspace $V \subset L^{2}(\Sigma)$ with $\operatorname{dim}(V) \leq d\left(q_{2}\right)$ such that for all $g \in V^{\perp}$

$$
\int_{\Omega}\left(\left|\nabla\left(u_{2}^{(g)}-u_{1}^{(g)}\right)\right|^{2}-k^{2} q_{2}\left|u_{2}^{(g)}-u_{1}^{(g)}\right|^{2}\right) \mathrm{d} x \geq 0 .
$$

Proof. Using Lemma 2.1, we have

$$
\int_{\Omega}\left(\left|\nabla\left(u_{2}^{(g)}-u_{1}^{(g)}\right)\right|^{2}-k^{2} q_{2}\left|u_{2}^{(g)}-u_{1}^{(g)}\right|^{2}\right) \mathrm{d} x \geq 0
$$

for all $g \in L^{2}(\Sigma)$ with $u_{2}^{(g)}-u_{1}^{(g)} \in V\left(q_{2}\right)^{\perp}$. The solution operators

$$
S_{j}: L^{2}(\Sigma) \rightarrow H^{1}(\Omega), \quad g \mapsto u_{j}^{(g)}, \quad \text { where } u_{j}^{(g)} \in H^{1}(\Omega) \text { solves }(2), \quad j \in\{1,2\},
$$

are linear and bounded, and

$$
\left(S_{2}-S_{1}\right) g=u_{2}^{(g)}-u_{1}^{(g)} \in V\left(q_{2}\right)^{\perp} \quad \text { if and only if } \quad g \in\left(\left(S_{2}-S_{1}\right)^{*} V\left(q_{2}\right)\right)^{\perp} .
$$

Since $\operatorname{dim}\left(S_{2}-S_{1}\right)^{*} V\left(q_{2}\right) \leq \operatorname{dim} V\left(q_{2}\right)=d\left(q_{2}\right)$, the assertion follows with $V:=\left(S_{2}-S_{1}\right)^{*} V\left(q_{2}\right)$. 
Proof of Theorem 3.5. This now immediately follows from combining Lemmas 3.7 and 3.8.

3C. The number of negative eigenvalues. We will now further investigate the number $d(q) \in \mathbb{N}_{0}$ (defined in Lemma 2.1) that bounds the number of negative eigenvalues in the monotonicity relations derived in Section 3B. We will show that $d(q)$ depends monotonously on the scattering index $q$ and show that $d(q)$ is less than or equal to the number of Neumann eigenvalues for the Laplacian which are larger than $-k^{2} q_{\max }$, where $q_{\max } \geq q(x)$ for all $x \in \Omega$ (a.e.).

Lemma 3.9. Let $q_{1}, q_{2} \in L^{\infty}(\Omega)$. Then $q_{1} \leq q_{2}$ implies $d\left(q_{1}\right) \leq d\left(q_{2}\right)$.

Proof. The inequality $q_{1} \leq q_{2}$ implies that $K_{q_{1}} \leq K_{q_{2}}$. Hence, the assertion follows from the equivalence of (a) and (c) in Corollary 3.3.

Lemma 3.10. Let $q \in L^{\infty}(\Omega)$, and $k \in \mathbb{R}$.

(a) There is a countable and discrete set of real values

$$
\lambda_{1} \geq \lambda_{2} \geq \lambda_{3} \cdots \rightarrow-\infty
$$

(called Neumann eigenvalues) such that

$$
\left(\Delta+k^{2} q\right) u=\lambda u \quad \text { in } \Omega,\left.\quad \partial_{\nu} u\right|_{\partial \Omega}=0,
$$

admits a nontrivial solution (called a Neumann eigenfunction) $0 \neq \equiv \in \in H^{1}(\Omega)$ if and only if $\lambda \in$ $\left\{\lambda_{1}, \lambda_{2}, \ldots\right\}$, and there is an orthonormal basis $\left(u_{1}, u_{2}, \ldots\right)$ of $L^{2}(\Omega)$ such that $u_{j} \in H^{1}(\Omega)$ is a Neumann eigenfunction for $\lambda_{j}$.

(b) If $\lambda$ is not a Neumann eigenvalue, then the problem

$$
\left(\Delta+k^{2} q\right) u=\lambda u+F \quad \text { in } \Omega,\left.\quad \partial_{\nu} u\right|_{\partial \Omega}=g,
$$

has a unique solution $u \in H^{1}(\Omega)$ for any $F \in L^{2}(\Omega)$ and $g \in L^{2}(\partial \Omega)$, and the solution operator is linear and bounded.

(c) Let $N_{+}:=\operatorname{span}\left\{u_{j}: \lambda_{j}>0\right\}$. Then $\operatorname{dim}\left(N_{+}\right)<\infty$,

$$
N_{-}:=\overline{\operatorname{span}\left\{u_{j}: \lambda_{j} \leq 0\right\}}=\left\{v \in H^{1}(\Omega): v \perp_{L^{2}} N_{+}\right\}
$$

is a complement of $N_{+}\left(\right.$in $\left.H^{1}(\Omega)\right)$, and

$$
\begin{array}{ll}
\int_{\Omega}|\nabla v|^{2}-k^{2} q v^{2} \mathrm{~d} x<0 & \text { for all } v \in N_{+}, \\
\int_{\Omega}|\nabla v|^{2}-k^{2} q v^{2} \mathrm{~d} x \geq 0 & \text { for all } v \in N_{-},
\end{array}
$$

where the closure in (14) is taken with respect to the $H^{1}(\Omega)$-norm, and $\perp_{L^{2}}$ denotes orthogonality with respect to the $L^{2}$ inner product.

(d) $d(q)$ is the number of positive Neumann eigenvalues of $\Delta+k^{2} q$; i.e., $d(q)=\operatorname{dim}\left(N_{+}\right)$.

(e) 0 is a Neumann eigenvalue if and only if $k>0$ is a resonance frequency. 
Proof. (a) Define $c:=k^{2}\|q\|_{L^{\infty}(\Omega)}+1>0$ and $R:=I-K-k^{2} K_{q}+c K$. Then $R$ is coercive and thus continuously invertible. Using the equivalent variational formulation of (12), we have that $\lambda \in \mathbb{R}$ is a Neumann eigenvalue with Neumann eigenfunction $u \neq \equiv 0$ if and only if

$$
\int_{\Omega}\left(-\nabla u \cdot \nabla v+k^{2} q u v\right) \mathrm{d} x=\lambda \int_{\Omega} u v \mathrm{~d} x \quad \text { for all } v \in H^{1}(\Omega),
$$

which is equivalent to

$$
\left(I-K-k^{2} K_{q}\right) u=-\lambda K u
$$

and thus to

$$
R u=\left(I-K-k^{2} K_{q}+c K\right) u=(c-\lambda) K u .
$$

This shows that $c$ cannot be a Neumann eigenvalue since $R u \not \equiv 0$ for $u \not \equiv 0$. Moreover, using $K=j^{*} j$, the invertibility of $R$, and the injectivity of $j$, we have that (17) is equivalent to

$$
\frac{1}{c-\lambda}(j u)=j R^{-1} j^{*}(j u) \text {. }
$$

This shows that $\lambda \in \mathbb{R}$ is a Neumann eigenvalue with Neumann eigenfunction $u \in H^{1}(\Omega)$ if and only if $j u \in L^{2}(\Omega)$ is an eigenfunction of $j R^{-1} j^{*}: L^{2}(\Omega) \rightarrow L^{2}(\Omega)$ with eigenvalue $1 /(c-\lambda)$. Since $j$ is injective, and every eigenfunction of $j R^{-1} j^{*}$ lies in the range of $j$, this is a one-to-one correspondence, and the dimension of the corresponding eigenspaces is the same. Since $j R^{-1} j^{*}$ is a compact, self-adjoint, positive operator, the assertions in (a) follow from the spectral theorem on self-adjoint compact operators.

(b) This follows from the fact that $I-K-k^{2} K_{q}-\lambda K$ is Fredholm of index 0 and thus continuously invertible if it is injective.

(c) $\operatorname{dim}\left(N_{+}\right)<\infty$ follows from (a). We define

$$
N_{-}:=\overline{\operatorname{span}\left\{u_{j}: \lambda_{j} \leq 0\right\}} \quad \text { and } \quad \tilde{N}_{-}:=\left\{v \in H^{1}(\Omega): v \perp_{L^{2}} N_{+}\right\} .
$$

$\widetilde{N}_{-}$is closed with respect to the $H^{1}$-norm and contains all $u_{j}$ with $\lambda_{j} \leq 0$, so that $N_{-} \subseteq \widetilde{N}_{-}$. To show $N_{-}=\widetilde{N}_{-}$, we argue by contradiction. If $N_{-} \subsetneq \widetilde{N}_{-}$, then there would exist a $0 \neq v \in \widetilde{N}_{-}$with $\left\langle u_{j}, v\right\rangle=0$ for all $u_{j}$ with $\lambda_{j} \leq 0$. Using

$$
\begin{aligned}
0 & =\left\langle u_{j}, v\right\rangle=\int_{\Omega}\left(\nabla u_{j} \cdot \nabla v+u_{j} v\right) \mathrm{d} x \\
& =\int_{\Omega}\left(\nabla u_{j} \cdot \nabla v-k^{2} q u_{j} v\right) \mathrm{d} x+\int_{\Omega}\left(1+k^{2} q\right) u_{j} v \mathrm{~d} x \\
& =\int_{\Omega}\left(1+k^{2} q-\lambda_{j}\right) u_{j} v \mathrm{~d} x,
\end{aligned}
$$

and the fact that $\lambda_{j} \rightarrow-\infty$, it would follow that $v \perp_{L^{2}} u_{j}$ for all but finitely many $u_{j}$. Since $v \perp_{L^{2}} N_{+}$, and $\left(u_{1}, u_{2}, \ldots\right)$ is an orthonormal basis of $L^{2}(\Omega), v$ must then be a finite combination of $u_{j}$ with $\lambda_{j} \leq 0$, which would imply that $v=0$. Hence, $N_{-}=\widetilde{N}_{-}$, so that the equality in (14) is proven. 
Obviously, $N_{+} \cap N_{-}=0$ and every $v \in H^{1}(\Omega)$ can be written as

$$
v=\sum_{\lambda_{j}>0}\left(\int_{\Omega} v u_{j} \mathrm{~d} x\right) u_{j}+\left(v-\sum_{\lambda_{j}>0}\left(\int_{\Omega} v u_{j} \mathrm{~d} x\right) u_{j}\right) \in N_{+}+N_{-},
$$

which shows that $N_{-}$is a complement of $N_{+}$.

To show (15), we use the $L^{2}$-orthogonality of the $u_{j}$ to obtain for all $v=\sum_{\lambda_{j}>0} \alpha_{j} u_{j} \in N_{+}$

$$
\begin{aligned}
\int_{\Omega}\left(|\nabla v|^{2}-k^{2} q v v\right) \mathrm{d} x & =\sum_{\lambda_{j}>0} \alpha_{j} \int_{\Omega}\left(\nabla u_{j} \cdot \nabla v-k^{2} q u_{j} v\right) \mathrm{d} x \\
& =-\sum_{\lambda_{j}>0} \alpha_{j} \lambda_{j} \int_{\Omega} u_{j} v \mathrm{~d} x=-\sum_{\lambda_{j}>0} \alpha_{j}^{2} \lambda_{j} \int_{\Omega} u_{j}^{2} \mathrm{~d} x<0 .
\end{aligned}
$$

Since every $v \in N_{-}$is an $H^{1}(\Omega)$-limit of finite linear combinations of $u_{j}$ with $\lambda_{j} \leq 0$, (16) follows with the same argument.

(d) Inequality (15) can be written as

$$
\left\langle\left(K+k^{2} K_{q}\right) v, v\right\rangle>\|v\|^{2} \quad \text { for all } v \in N_{+} .
$$

Lemma 3.2(b) implies that the number $d(q)$ of eigenvalues of $K+k^{2} K_{q}$ larger than 1 must be at least $\operatorname{dim}\left(N_{+}\right)$. Likewise, (16) can be written as

$$
\left\langle\left(K+k^{2} K_{q}\right) v, v\right\rangle \leq\|v\|^{2} \quad \text { for all } v \in N_{-} .
$$

Hence, Lemma 3.2(a) shows that $d(q)$ is at $\operatorname{most} \operatorname{codim}\left(N_{-}\right)=\operatorname{dim}\left(N_{+}\right)$.

(e) This is trivial.

Corollary 3.11. If $q \in L^{\infty}(\Omega)$ and $q(x) \leq q_{\max } \in \mathbb{R}$ for all $x \in \Omega$ (a.e.), then $d(q) \leq d\left(q_{\max }\right)$, and $d\left(q_{\max }\right)$ is the number of Neumann eigenvalues of the Laplacian $\Delta$ that are larger than $-k^{2} q_{\max }$.

Proof. Obviously, the number of positive Neumann eigenvalues of $\Delta+k^{2} q_{\max }$ agrees with the number of Neumann eigenvalues of the Laplacian $\Delta$ that are greater than $-k^{2} q_{\max }$. Hence, the assertion follows from Lemmas 3.9 and 3.10(d).

Remark 3.12. One can show, by using constant potentials, that for the Helmholtz equation $\Lambda_{q_{2}}-\Lambda_{q_{1}}$ can actually have negative eigenvalues when $q_{1} \leq q_{2}$. This shows that in Theorem 3.5 it is indeed necessary to work modulo a finite-dimensional subspace. The details will appear in a subsequent work.

\section{Localized potentials for the Helmholtz equation}

We now extend the result in [Gebauer 2008] to the Helmholtz equation and prove that we can control the energy terms appearing in the monotonicity relation in spaces of finite codimension. We will first state the result and prove it using a functional analytic relation between operator norms and the ranges of their adjoints in Section 4A. Section 4B then gives an alternative proof that is based on a Runge approximation argument. 
4A. Localized potentials. Our main result on controlling the solutions of the Helmholtz equation in spaces of finite codimension is the following theorem.

Theorem 4.1. Let $q \in L^{\infty}(\Omega) \backslash\{0\}$ for which $k>0$ is not a resonance. Let $B, D \subseteq \bar{\Omega}$ be measurable, $B \backslash \bar{D}$ possess positive measure, and $\bar{\Omega} \backslash \bar{D}$ be connected to $\Sigma$.

Then for any subspace $V \subset L^{2}(\Sigma)$ with $\operatorname{dim} V<\infty$, there exists a sequence $\left(g_{j}\right)_{j \in \mathbb{N}} \subset V^{\perp}$ such that

$$
\int_{B}\left|u_{q}^{\left(g_{j}\right)}\right|^{2} \mathrm{~d} x \rightarrow \infty \text { and } \int_{D}\left|u_{q}^{\left(g_{j}\right)}\right|^{2} \mathrm{~d} x \rightarrow 0,
$$

where $u_{q}^{\left(g_{j}\right)} \in H^{1}(\Omega)$ solves the Helmholtz equation (2) with Neumann boundary data $g_{j}$.

The arguments that we will use to prove Theorem 4.1 in this subsection also yield a simple proof for the following elementary result. We formulate it as a theorem since we will utilize it in the next section to control energy terms in monotonicity inequalities for different scattering coefficients.

Theorem 4.2. Let $q_{1}, q_{2} \in L^{\infty}(\Omega) \backslash\{0\}$ for which $k>0$ is not a resonance. If $q_{1}(x)=q_{2}(x)$ for all $x$ (a.e.) outside a measurable set $D \subset \Omega$, then there exist constants $c_{1}, c_{2}>0$ such that

$$
c_{1} \int_{D}\left|u_{1}^{(g)}\right|^{2} \mathrm{~d} x \leq \int_{D}\left|u_{2}^{(g)}\right|^{2} \mathrm{~d} x \leq c_{2} \int_{D}\left|u_{1}^{(g)}\right|^{2} \mathrm{~d} x \quad \text { for all } g \in L^{2}(\Sigma),
$$

where $u_{1}^{(g)}, u_{2}^{(g)} \in H^{1}(\Omega)$ solve the Helmholtz equation (2) with Neumann boundary data $g$ and $q=q_{1}$, $q=q_{2}$ respectively.

To prove Theorems 4.1 and 4.2 we will formulate and prove several lemmas. Let us first note that the assertion of Theorem 4.1 already holds if we can prove it for a subset of $B$ with positive measure. We will use the subset $B \cap C$, where $C$ is a small closed ball constructed in the next lemma.

Lemma 4.3. Let $B, D \subseteq \bar{\Omega}$ be measurable, $B \backslash \bar{D}$ possess positive measure, and $\bar{\Omega} \backslash \bar{D}$ be connected to $\Sigma$. Then there exists a closed ball $C$ such that $B \cap C$ has positive measure, $C \cap \bar{D}=\varnothing$, and $\bar{\Omega} \backslash(\bar{D} \cup C)$ is connected to $\Sigma$.

Proof. Let $x$ be a point of Lebesgue density 1 in $B \backslash \bar{D}$. Then the closure $C$ of a sufficiently small ball centered in $x$ will satisfy $B \cap C$ has positive measure, $C \cap \bar{D}=\varnothing$, and $\bar{\Omega} \backslash(\bar{D} \cup C)$ is connected to $\Sigma$.

Now we follow the general approach in [Gebauer 2008]. We formulate the energy terms in Theorem 4.1 as norms of operator evaluations and characterize their adjoints. Then we characterize the ranges of the adjoints using the unique continuation property, and prove Theorem 4.1 using a functional-analytic relation between norms of operator evaluations and ranges of their adjoints.

Lemma 4.4. Let $q \in L^{\infty}(\Omega) \backslash\{0\}$ for which $k>0$ is not a resonance. For a measurable set $D \subset \Omega$ we define

$$
L_{D}: L^{2}(\Sigma) \rightarrow L^{2}(D),\left.\quad g \mapsto u\right|_{D},
$$

where $u \in H^{1}(\Omega)$ solves (2). Then $L_{D}$ is a compact linear operator, and its adjoint satisfies

$$
L_{D}^{*}: L^{2}(D) \rightarrow L^{2}(\Sigma),\left.\quad f \mapsto v\right|_{\Sigma}
$$


where $v$ solves

$$
\Delta v+k^{2} q v=f \chi_{D},\left.\quad \partial_{\nu} v\right|_{\partial \Omega}=0 .
$$

Proof. With the operators $I, j$, and $K_{q}$ defined as in Section 2A and (4) we have

$$
L_{D}=R_{D} j\left(I-K-k^{2} K_{q}\right)^{-1} \gamma_{\Sigma}^{*},
$$

where $R_{D}: L^{2}(\Omega) \rightarrow L^{2}(D)$ is the restriction operator $\left.v \rightarrow v\right|_{D}$. Hence, $L_{D}$ is a linear compact operator, and its adjoint is

$$
L_{D}^{*}=\gamma_{\Sigma}\left(I-K-k^{2} K_{q}\right)^{-1} j^{*} R_{D}^{*}
$$

Thus $L_{D}^{*} f=\left.v\right|_{\Sigma}$, where $v \in H^{1}(\Omega)$ solves

$$
\left(I-K-k^{2} K_{q}\right) v=j^{*} R_{D}^{*} f
$$

i.e., for all $w \in H^{1}(\Omega)$,

$$
\int_{\Omega}\left(\nabla v \cdot \nabla w-k^{2} q v w\right) \mathrm{d} x=\left\langle\left(I-K-k^{2} K_{q}\right) v, w\right\rangle=\left\langle j^{*} R_{D}^{*} f, w\right\rangle=\int_{D} f w \mathrm{~d} x,
$$

which is the variational formulation equivalent to (18).

Lemma 4.5. Let $q \in L^{\infty}(\Omega) \backslash\{0\}$ for which $k>0$ is not a resonance. Let $B, D \subseteq \bar{\Omega}$ be measurable and $C \subseteq \bar{\Omega}$ be a closed set such that $B \cap C$ has positive measure, $C \cap \bar{D}=\varnothing$, and $\bar{\Omega} \backslash(\bar{D} \cup C)$ is connected to $\Sigma$. Then,

$$
\mathcal{R}\left(L_{B \cap C}^{*}\right) \cap \mathcal{R}\left(L_{D}^{*}\right)=\{0\},
$$

and $\mathcal{R}\left(L_{B \cap C}^{*}\right), \mathcal{R}\left(L_{D}^{*}\right) \subset L^{2}(\Sigma)$ are both dense (and thus in particular infinite-dimensional).

Proof. It follows from the unique continuation property in Theorem 2.4(a) that $L_{B \cap C}$ and $L_{D}$ are injective. Hence $\mathcal{R}\left(L_{B \cap C}^{*}\right)$ and $\mathcal{R}\left(L_{D}^{*}\right)$ are dense subspaces of $L^{2}(\Sigma)$.

The characterization of the adjoint operators in Lemma 4.4 shows that

$$
B \cap C \subseteq C \text { implies } \mathcal{R}\left(L_{B \cap C}^{*}\right) \subseteq \mathcal{R}\left(L_{C}^{*}\right) .
$$

Hence, (19) follows a fortiori if we can show that

$$
\mathcal{R}\left(L_{C}^{*}\right) \cap \mathcal{R}\left(L_{D}^{*}\right)=\{0\} .
$$

To show this let $h \in \mathcal{R}\left(L_{C}^{*}\right) \cap \mathcal{R}\left(L_{D}^{*}\right)$. Then there exist $f_{C} \in L^{2}(C), f_{D} \in L^{2}(D)$, and $v_{C}, v_{D} \in H^{1}(\Omega)$ such that

$$
\begin{aligned}
\Delta v_{C}+k^{2} q v_{C}=f_{C} \chi_{C}, & \left.\partial_{\nu} v\right|_{\partial \Omega}=0, \\
\Delta v_{D}+k^{2} q v_{D}=f_{D} \chi_{D}, & \left.\partial_{\nu} v\right|_{\partial \Omega}=0,
\end{aligned}
$$

and $\left.v_{C}\right|_{\Sigma}=h=\left.v_{D}\right|_{\Sigma}$.

It follows from the unique continuation property in Theorem 2.4(b) that $v_{C}=v_{D}$ on the connected set $\Omega \backslash(C \cup \bar{D})$. Hence,

$$
v:= \begin{cases}v_{C}=v_{D} & \text { on } \Omega \backslash(C \cup \bar{D}), \\ v_{C} & \text { on } \bar{D}, \\ v_{D} & \text { on } C\end{cases}
$$


defines an $H^{1}(\Omega)$-function solving

$$
\Delta v+k^{2} q v=0,\left.\quad \partial_{\nu} v\right|_{\partial \Omega}=0,
$$

so that $v=0$ and thus $h=\left.v_{C}\right|_{\Sigma}=\left.v_{D}\right|_{\Sigma}=\left.v\right|_{\Sigma}=0$.

Lemma 4.6. Let $X, Y$ and $Z$ be Hilbert spaces, and $A_{1}: X \rightarrow Y$ and $A_{2}: X \rightarrow Z$ be linear bounded operators. Then

$$
\exists c>0:\left\|A_{1} x\right\| \leq c\left\|A_{2} x\right\| \quad \forall x \in X \quad \text { if and only if } \quad \mathcal{R}\left(A_{1}^{*}\right) \subseteq \mathcal{R}\left(A_{2}^{*}\right) .
$$

Proof. This is proven for reflexive Banach spaces in [Gebauer 2008, Lemma 2.5]. Note that one direction of the implication also holds in nonreflexive Banach spaces; see [Gebauer 2008, Lemma 2.4].

Lemma 4.7. Let $V, X, Y \subset Z$ be subspaces of a real vector space $Z$. If

$$
X \cap Y=\{0\} \text { and } X \subseteq Y+V,
$$

then $\operatorname{dim}(X) \leq \operatorname{dim}(V)$.

Proof. Let $\left(x_{j}\right)_{j=1}^{m} \subset X$ be a linearly independent sequence of $m$ vectors. Then there exist $\left(y_{j}\right)_{j=1}^{m} \subset Y$ and $\left(v_{j}\right)_{j=1}^{m} \subset V$ such that $x_{j}=y_{j}+v_{j}$ for all $j=1, \ldots, m$. We will prove the assertion by showing that the sequence $\left(v_{j}\right)_{j=1}^{m}$ is linearly independent. To this end let $\sum_{j=1}^{m} a_{j} v_{j}=0$ with $a_{j} \in \mathbb{R}, j=1, \ldots, m$. Then

$$
\sum_{j=1}^{m} a_{j} x_{j}=\sum_{j=1}^{m} a_{j}\left(y_{j}+v_{j}\right)=\sum_{j=1}^{m} a_{j} y_{j} \in Y,
$$

so that $\sum_{j=1}^{m} a_{j} x_{j}=0$. Since $\left(x_{j}\right)_{j=1}^{m} \subset X$ is linearly independent, it follows that $a_{j}=0$ for all $j=1, \ldots, m$. This shows that $\left(v_{j}\right)_{j=1}^{m}$ is linearly independent.

Proof of Theorem 4.1. Let $q \in L^{\infty}(\Omega) \backslash\{0\}$ for which $k>0$ is not a resonance. Let $B, D \subseteq \bar{\Omega}$ be measurable, $B \backslash \bar{D}$ possess positive measure, and $\bar{\Omega} \backslash \bar{D}$ be connected to $\Sigma$. Using Lemma 4.3 we obtain a closed set $C \subseteq \bar{\Omega}$ such that $B \cap C$ has positive measure, $C \cap \bar{D}=\varnothing$, and $\bar{\Omega} \backslash(\bar{D} \cup C)$ is connected to $\Sigma$.

Let $V \subset L^{2}(\Sigma)$ be a subspace with $d:=\operatorname{dim}(V)<\infty$. Since $V$ is finite-dimensional and thus closed, there exists an orthogonal projection operator $P_{V}: L^{2}(\Sigma) \rightarrow L^{2}(\Sigma)$ with

$$
\mathcal{R}\left(P_{V}\right)=V, \quad P_{V}^{2}=P_{V}, \quad \text { and } \quad P_{V}=P_{V}^{*} .
$$

From Lemma 4.5, we have that $\mathcal{R}\left(L_{B \cap C}^{*}\right) \cap \mathcal{R}\left(L_{D}^{*}\right)=0$ and that $\mathcal{R}\left(L_{B \cap C}^{*}\right)$ is infinite-dimensional. So it follows from Lemma 4.7 that

$$
\mathcal{R}\left(L_{B \cap C}^{*}\right) \nsubseteq \mathcal{R}\left(L_{D}^{*}\right)+V=\mathcal{R}\left(L_{D}^{*}\right)+\mathcal{R}\left(P_{V}^{*}\right) .
$$

Since $B \cap C \subseteq B$ implies that $\mathcal{R}\left(L_{B \cap C}^{*}\right) \subseteq \mathcal{R}\left(L_{B}^{*}\right)$, and since (using block operator matrix notation)

$$
\mathcal{R}\left(\left(L_{D}^{*} P_{V}^{*}\right)\right) \subseteq \mathcal{R}\left(L_{D}^{*}\right)+\mathcal{R}\left(P_{V}^{*}\right),
$$

we obtain that

$$
\mathcal{R}\left(L_{B}^{*}\right) \nsubseteq \mathcal{R}\left(\left(L_{D}^{*} \quad P_{V}^{*}\right)\right)=\mathcal{R}\left(\left(\begin{array}{c}
L_{D} \\
P_{V}
\end{array}\right)^{*}\right)
$$


It then follows from Lemma 4.6 that there cannot exist a constant $C>0$ with

$$
\left\|L_{B} g\right\|^{2} \leq C^{2}\left\|\left(\begin{array}{c}
L_{D} \\
P_{V}
\end{array}\right) g\right\|^{2}=C^{2}\left\|L_{D} g\right\|^{2}+C^{2}\left\|P_{V} g\right\|^{2} \quad \text { for all } g \in L^{2}(\Sigma) .
$$

Hence, there must exist a sequence $\left(\tilde{g}_{k}\right)_{k \in \mathbb{N}} \subseteq L^{2}(\Sigma)$ with

$$
\left\|L_{B} \tilde{g}_{k}\right\| \rightarrow \infty \quad \text { and } \quad\left\|L_{D} \tilde{g}_{k}\right\|,\left\|P_{V} \tilde{g}_{k}\right\| \rightarrow 0 .
$$

Thus, $g_{k}:=\tilde{g}_{k}-P_{V} \tilde{g}_{k} \in V^{\perp} \subseteq L^{2}(\Sigma)$ and

$$
\left\|L_{B} g_{k}\right\| \geq\left\|L_{B} \tilde{g}_{k}\right\|-\left\|L_{B}\right\|\left\|P_{V} \tilde{g}_{k}\right\| \rightarrow \infty \quad \text { and } \quad\left\|L_{D} g_{k}\right\| \rightarrow 0,
$$

which shows the assertion.

Proof of Theorem 4.2. Let $q_{1}, q_{2} \in L^{\infty}(\Omega)$ for which $k>0$ is not a resonance, and let $q_{1}(x)=q_{2}(x)$ for all $x$ (a.e.) outside a measurable set $D \subset \Omega$. We denote by $L_{q_{1}, D}$ and $L_{q_{2}, D}$ the operators from Lemma 4.4 for $q=q_{1}$ and $q=q_{2}$. For $f \in L^{2}(D)$, we then have

where $v_{1}, v_{2} \in H^{1}(\Omega)$ solve

$$
L_{q_{1}, D}^{*} f=\left.v_{1}\right|_{\Sigma} \quad \text { and } \quad L_{q_{2}, D}^{*} f=\left.v_{2}\right|_{\Sigma}
$$

Since this also implies

$$
\begin{array}{ll}
\Delta v_{1}+k^{2} q_{1} v_{1}=f \chi_{D}, & \left.\partial_{\nu} v_{1}\right|_{\partial \Omega}=0 \\
\Delta v_{2}+k^{2} q_{2} v_{2}=f \chi_{D}, & \left.\partial_{\nu} v_{2}\right|_{\partial \Omega}=0 .
\end{array}
$$

$$
\begin{array}{ll}
\Delta v_{1}+k^{2} q_{2} v_{1}=f \chi_{D}+k^{2}\left(q_{2}-q_{1}\right) v_{1}, & \left.\partial_{\nu} v_{1}\right|_{\partial \Omega}=0, \\
\Delta v_{2}+k^{2} q_{1} v_{2}=f \chi_{D}+k^{2}\left(q_{1}-q_{2}\right) v_{2}, & \left.\partial_{\nu} v_{2}\right|_{\partial \Omega}=0,
\end{array}
$$

and $q_{1}-q_{2}$ vanishes (a.e.) outside $D$, it follows that

$$
\left.v_{1}\right|_{\Sigma}=L_{q_{2}, D}^{*}\left(f+k^{2}\left(q_{2}-q_{1}\right) v_{1}\right) \quad \text { and }\left.\quad v_{2}\right|_{\Sigma}=L_{q_{1}, D}^{*}\left(f+k^{2}\left(q_{1}-q_{2}\right) v_{2}\right) .
$$

Hence, $\mathcal{R}\left(L_{q_{1}, D}^{*}\right)=\mathcal{R}\left(L_{q_{2}, D}^{*}\right)$, so that the assertion follows from Lemma 4.6.

4B. Localized potentials and Runge approximation. In this subsection we give an alternative proof of Theorem 4.1 that is based on a Runge approximation argument that characterizes whether a given function $\varphi \in L^{2}(O)$ on a measurable subset $O \subseteq \Omega$ can be approximated by functions in a subspace of solutions of the Helmholtz equation in $\Omega$. Throughout this subsection let $q \in L^{\infty}(\Omega) \backslash\{0\}$ for which $k>0$ is not a resonance. We will prove the following theorem.

Theorem 4.8. Let $D \subseteq \Omega$ be a measurable set and $C \subset \Omega$ be a closed ball for which $C \cap \bar{D}=\varnothing$, and $\bar{\Omega} \backslash(C \cup \bar{D})$ is connected to $\Sigma$.

Then for any subspace $V \subset L^{2}(\Sigma)$ with $\operatorname{dim} V<\infty$, there exists a function $\varphi \in L^{2}(C \cup \bar{D})$ that can be approximated (in the $L^{2}(C \cup \bar{D})$-norm) by solutions $u \in H^{1}(\Omega)$ of

$$
\left(\Delta+k^{2} q\right) u=0 \quad \text { in } \Omega \quad \text { with }\left.\quad \partial_{\nu} u\right|_{\partial \Omega \backslash \Sigma}=0,\left.\quad \partial_{\nu} u\right|_{\Sigma} \in V^{\perp},
$$


and satisfies

$$
\left.\varphi\right|_{\bar{D}} \equiv 0 \quad \text { and }\left.\quad \varphi\right|_{B} \not \equiv 0
$$

for all subsets $B \subseteq C$ with positive measure.

Before we prove Theorem 4.8, let us first show that it implies Theorem 4.1.

Corollary 4.9. Let $B, D \subseteq \bar{\Omega}$ be measurable, $B \backslash \bar{D}$ possess positive measure, and $\bar{\Omega} \backslash \bar{D}$ be connected to $\Sigma$. Then for any subspace $V \subset L^{2}(\Sigma)$ with $\operatorname{dim} V<\infty$, there exists a sequence $\left(g_{j}\right)_{j \in \mathbb{N}} \subset V^{\perp}$ such that

$$
\int_{B}\left|u_{q}^{\left(g_{j}\right)}\right|^{2} \mathrm{~d} x \rightarrow \infty \text { and } \quad \int_{D}\left|u_{q}^{\left(g_{j}\right)}\right|^{2} \mathrm{~d} x \rightarrow 0,
$$

where $u_{q}^{\left(g_{j}\right)} \in H^{1}(\Omega)$ solves the Helmholtz equation (2) with Neumann boundary data $g_{j}$.

Proof. As in Lemma 4.3, we can find a closed ball $C \subset \Omega$ so that $B \cap C$ has positive measure, $C \cap \bar{D}=\varnothing$, and $\bar{\Omega} \backslash(\bar{D} \cup C)$ is connected to $\Sigma$. Using Theorem 4.8, there exists $\varphi \in L^{2}(C \cup \bar{D})$ and a sequence of solutions $\left(\tilde{u}^{(j)}\right)_{j \in \mathbb{N}} \subset H^{1}(\Omega)$ of $\left(\Delta+k^{2} q\right) \tilde{u}^{(j)}=0$ in $\Omega$ with $\left.\partial_{\nu} \tilde{u}^{(j)}\right|_{\partial \Omega \backslash \Sigma}=0,\left.\partial_{\nu} \tilde{u}^{(j)}\right|_{\Sigma} \in V^{\perp}$,

$$
\left\|\left.\tilde{u}^{(j)}\right|_{B \cap C}\right\|_{L^{2}(B \cap C)} \rightarrow\|\varphi\|_{L^{2}(B \cap C)}>0 \quad \text { and } \quad\left\|\left.\tilde{u}^{(j)}\right|_{\bar{D}}\right\|_{L^{2}(\bar{D})} \rightarrow 0 .
$$

Obviously, the scaled sequence

satisfies the assertion.

$$
g^{(j)}:=\frac{\partial_{\nu} \tilde{u}^{(j)}}{\sqrt{\left\|\left.\tilde{u}^{(j)}\right|_{\bar{D}}\right\|_{L^{2}(\bar{D})}}} \in V^{\perp}
$$

To prove Theorem 4.8, we start with an abstract characterization showing whether a given function $\varphi \in L^{2}(O)$ on a measurable set $O \subseteq \Omega$ is a limit of functions from a subspace of solutions of the Helmholtz equation in $\Omega$. For the sake of readability, we write $v \chi_{O} \in L^{2}(\Omega)$ for the zero extension of a function $v \in L^{2}(O)$, and we write the dual pairing on $H^{-1 / 2}(\partial \Omega) \times H^{1 / 2}(\partial \Omega)$ as an integral over $\partial \Omega$.

Lemma 4.10. Let $O \subseteq \Omega$ be measurable. Let $H \subseteq H^{1}(\Omega)$ be a (not necessarily closed) subspace of solutions of $\left(\Delta+k^{2} q\right) u=0$ in $\Omega$.

A function $\varphi \in L^{2}(O)$ can be approximated on $O$ by solutions $u \in H$ in the sense that

$$
\inf _{u \in H}\|\varphi-u\|_{L^{2}(O)}=0
$$

if and only if $\int_{O} \varphi v \mathrm{~d} x=0$ for all $v \in L^{2}(O)$ for which the solution $w \in H^{1}(\Omega)$ of

$$
\left(\Delta+k^{2} q\right) w=v \chi_{O} \quad \text { and }\left.\quad \partial_{\nu} w\right|_{\partial \Omega}=0
$$

satisfies $\left.\left.\int_{\partial \Omega} \partial_{\nu} u\right|_{\partial \Omega} w\right|_{\partial \Omega} \mathrm{d} s=0$ for all $u \in H$.

Proof. Let

$$
\mathcal{R}:=\left\{\left.u\right|_{O}: u \in H\right\} \subseteq L^{2}(O) .
$$

Let $v \in L^{2}(O)$ and $w \in H^{1}(\Omega)$ solve (20). Then $v \in \mathcal{R}^{\perp}$ if and only if, for all $u \in H$,

$0=\int_{O} u v \mathrm{~d} x=\int_{\Omega} u\left(\Delta+k^{2} q\right) w \mathrm{~d} x=\int_{\Omega} w\left(\Delta+k^{2} q\right) u \mathrm{~d} x-\left.\left.\int_{\partial \Omega} \partial_{\nu} u\right|_{\partial \Omega} w\right|_{\partial \Omega} \mathrm{d} s=-\left.\left.\int_{\partial \Omega} \partial_{\nu} u\right|_{\partial \Omega} w\right|_{\partial \Omega} \mathrm{d} s$. 
Hence, the assertion follows from $\overline{\mathcal{R}}=\left(\mathcal{R}^{\perp}\right)^{\perp}$ (where orthogonality and closures are taken with respect to the $L^{2}(O)$ inner product).

Now we characterize the functions $w$ appearing in Lemma 4.10 for a setting that will be considered in the proof of Theorem 4.8 .

Lemma 4.11. Let $V$ be a finite-dimensional subspace of $L^{2}(\Sigma)$, and $O \subset \Omega$ be a closed set for which the complement $\bar{\Omega} \backslash O$ is connected to $\Sigma$.

We define the spaces

$$
\begin{aligned}
W: & =\left\{w \in H^{1}(\Omega): \exists v \in L^{2}(O) \text { s.t. }\left(\Delta+k^{2} q\right) w=v \chi_{O},\left.\partial_{\nu} w\right|_{\partial \Omega}=0,\left.w\right|_{\Sigma} \in V\right\}, \\
W_{0}: & =\left\{w \in H^{1}(\Omega): \exists v \in L^{2}(O) \text { s.t. }\left(\Delta+k^{2} q\right) w=v \chi_{O},\left.\partial_{\nu} w\right|_{\partial \Omega}=0,\left.w\right|_{\Sigma}=0\right\} .
\end{aligned}
$$

Then the codimension $d:=\operatorname{dim}\left(W / W_{0}\right)$ of $W_{0}$ in $W$ is at most $\operatorname{dim}(V)$; i.e., there exist functions $w_{1}, \ldots, w_{d} \in W$ such that every $w \in W$ can be written as

$$
w=w_{0}+\sum_{j=1}^{d} a_{j} w_{j},
$$

with (w-dependent) $w_{0} \in W_{0}$ and $a_{1}, \ldots, a_{d} \in \mathbb{R}$.

Proof. $W_{0}$ is the kernel of the restricted trace operator

$$
\left.\gamma_{\Sigma}\right|_{W}: W \rightarrow V,\left.\quad w \mapsto w\right|_{\Sigma} .
$$

Hence, the codimension of $W_{0}$ as a subspace of $W$ is

$$
\operatorname{dim}\left(W / W_{0}\right)=\operatorname{dim}\left(\mathcal{R}\left(\left.\gamma_{\Sigma}\right|_{W}\right)\right) \leq \operatorname{dim}(V),
$$

which proves the assertion.

Proof of Theorem 4.8. Let $D \subseteq \Omega$ be a measurable set and $C \subset \Omega$ be a closed ball for which $C \cap \bar{D}=\varnothing$ and $\bar{\Omega} \backslash(C \cup \bar{D})$ is connected to $\Sigma$. Let $V$ be a finite-dimensional subspace of $L^{2}(\Sigma)$.

To apply Lemma 4.10, we set $O:=C \cup \bar{D}$ and

$$
H:=\left\{u \in H^{1}(\Omega):\left(\Delta+k^{2} q\right) u=0 \text { in } \Omega,\left.\partial_{\nu} u\right|_{\partial \Omega \backslash \Sigma}=0,\left.\partial_{\nu} u\right|_{\Sigma} \in V^{\perp}\right\} .
$$

Then $w \in H^{1}(\Omega)$ satisfies (20) and

$$
\left.\left.\int_{\partial \Omega} \partial_{\nu} u\right|_{\partial \Omega} w\right|_{\partial \Omega} \mathrm{d} s=0
$$

for all $u \in H$ if and only if $w \in W$, with $W$ defined in Lemma 4.11. Hence, by Lemma 4.10, a function $\varphi \in L^{2}(C \cup \bar{D})$ can be approximated by solutions $u \in H$ if and only if

$$
\int_{C \cup \bar{D}} \varphi\left(\Delta+k^{2} q\right) w \mathrm{~d} x=0 \quad \text { for all } w \in W .
$$

Thus, the assertion of Theorem 4.8 follows if we can show that there exists $\varphi \in L^{2}(C \cup \bar{D})$ that satisfies (21) and vanishes on $D$ but not on any subset of $C$ having positive measure. 
To construct such a $\varphi$, we first note that the Helmholtz equation (2) on $\Omega$ is uniquely solvable for all Neumann data $g \in L^{2}(\Sigma)$, and by unique continuation, linearly independent Neumann data yield solutions whose restrictions to the open ball $C^{\circ}$ are linearly independent. Hence, there exists an infinite number of linearly independent solutions

$$
\varphi_{j} \in H^{1}\left(C^{\circ}\right) \quad \text { with } \quad\left(\Delta+k^{2} q\right) \varphi_{j}=0 \quad \text { in } C^{\circ}, \quad j \in \mathbb{N} .
$$

We extend $\varphi_{j}$ by zero on $\bar{D} \cup \partial C$ to $\varphi_{j} \in L^{2}(O)$.

Every $w_{0} \in W_{0}$, with $W_{0}$ from Lemma 4.11, must possess zero Cauchy data $\left.w_{0}\right|_{\partial C}=0$ and $\left.\partial_{\nu} w_{0}\right|_{\partial C}=0$ by unique continuation. Hence, for all $w_{0} \in W_{0}$, and $j \in \mathbb{N}$,

$$
\int_{O} \varphi_{j}\left(\Delta+k^{2} q\right) w_{0} \mathrm{~d} x=\int_{C} \varphi_{j}\left(\Delta+k^{2} q\right) w_{0} \mathrm{~d} x=\int_{\partial C}\left(\left.\left.\varphi_{j}\right|_{\partial C} \partial_{\nu} w_{0}\right|_{\partial C}-\left.\left.\partial_{\nu} \varphi_{j}\right|_{\partial C} w_{0}\right|_{\partial C}\right) \mathrm{d} s=0 .
$$

Moreover, by a dimensionality argument, there must exist a nontrivial finite linear combination $\varphi$ of the infinitely many linearly independent $\varphi_{j}$ such that

$$
\int_{O} \varphi\left(\Delta+k^{2} q\right) w_{k} \mathrm{~d} x=0
$$

for the finitely many functions $w_{1}, \ldots, w_{d} \in W$ from Lemma 4.11. Thus, using Lemma 4.11, we have constructed a function $\varphi \in L^{2}(O)$ with $\left.\varphi\right|_{\bar{D}} \equiv 0,\left.\varphi\right|_{C^{\circ}} \not \equiv 0$, and

$$
\int_{O} \varphi\left(\Delta+k^{2} q\right) w \mathrm{~d} x=0 \quad \text { for all } w \in W=W_{0}+\operatorname{span}\left\{w_{1}, \ldots, w_{d}\right\} .
$$

Moreover, $\varphi$ solves (22), so that the unique continuation result from measurable sets in Theorem 2.4 also yields that $\left.\varphi\right|_{B} \not \equiv 0$ for all $B \subseteq C^{\circ}$ with positive measure. Since $\partial C$ is a null set, the latter also holds for all $B \subseteq C$ with positive measure. As explained above, the assertion of Theorem 4.8 now follows from Lemma 4.10.

\section{Local uniqueness for the Helmholtz equation}

We are now able to prove the first main result in this work, announced as Theorem 1.1 in the Introduction, and extend the local uniqueness result in [Harrach and Ullrich 2017] to the case of negative potentials and $n \geq 2$.

As in Section 2A, let $\Omega \subset \mathbb{R}^{n}, n \geq 2$, denote a bounded Lipschitz domain, and let $\Sigma \subseteq \partial \Omega$ be an arbitrarily small, relatively open part of the boundary $\partial \Omega$. For $q_{1}, q_{2} \in L^{\infty}(\Omega)$ let

$$
\Lambda\left(q_{1}\right), \Lambda\left(q_{2}\right): L^{2}(\Sigma) \rightarrow L^{2}(\Sigma), \quad \Lambda\left(q_{1}\right):\left.g \mapsto u_{1}\right|_{\Sigma}, \quad \Lambda\left(q_{2}\right):\left.g \mapsto u_{2}\right|_{\Sigma},
$$

be the Neumann-to-Dirichlet operators for the Helmholtz equation

$$
\left(\Delta+k^{2} q\right) u=0 \quad \text { in } \Omega,\left.\quad \partial_{\nu} u\right|_{\partial \Omega}= \begin{cases}g & \text { on } \Sigma, \\ 0 & \text { else, }\end{cases}
$$

with $q=q_{1}$ and $q=q_{2}$ respectively, and let $k>0$ be such that it is not a resonance for $q_{1}$ or $q_{2}$. 
Theorem 5.1. Let $q_{1} \leq q_{2}$ in a relatively open set $O \subseteq \bar{\Omega}$ that is connected to $\Sigma$. Then

$$
\left.\left.q_{1}\right|_{o} \not \equiv q_{2}\right|_{o} \quad \text { implies } \quad \Lambda\left(q_{1}\right) \neq \Lambda\left(q_{2}\right) .
$$

Moreover, in that case, $\Lambda\left(q_{2}\right)-\Lambda\left(q_{1}\right)$ has infinitely many positive eigenvalues.

Proof. If $q_{1}\left|O \not \equiv q_{2}\right|_{O}$ then there exists a subset $B \subseteq O$ with positive measure, and a constant $c>0$ such that $q_{2}(x)-q_{1}(x) \geq c$ for all $x \in B$ (a.e.). From the monotonicity inequality in Theorem 3.5 we have that $\Lambda\left(q_{2}\right)-\Lambda\left(q_{1}\right) \geq$ fin $A$, where

$$
A: L^{2}(\Sigma) \rightarrow L^{2}(\Sigma), \quad \int_{\Sigma} h A g \mathrm{~d} s=\int_{\Omega} k^{2}\left(q_{2}-q_{1}\right) u_{1}^{(g)} u_{1}^{(h)} \mathrm{d} x
$$

Note that $A=S_{1}^{*} j^{*} k^{2} M_{q_{1}-q_{2}} j S_{1}$, where $S_{1}: g \mapsto u_{1}^{(g)}$ is the solution operator and $j: H^{1}(\Omega) \rightarrow L^{2}(\Omega)$ is the compact inclusion, so $A$ is indeed a compact, self-adjoint linear operator on $L^{2}(\Sigma)$.

We will now prove the assertion by contradiction and assume that $\Lambda\left(q_{2}\right)-\Lambda\left(q_{1}\right) \leq$ fin 0 . Then, the transitivity result in Lemma 3.4 gives that $A \leq_{\text {fin }} 0$. By the characterization in Corollary 3.3, there would exist a finite-dimensional subspace $V \subseteq L^{2}(\partial \Omega)$, with

$$
\begin{aligned}
0 & \geq \int_{\Omega} k^{2}\left(q_{2}-q_{1}\right)\left|u_{1}^{(g)}\right|^{2} \mathrm{~d} x \\
& =\int_{O} k^{2}\left(q_{2}-q_{1}\right)\left|u_{1}^{(g)}\right|^{2} \mathrm{~d} x+\int_{\Omega \backslash O} k^{2}\left(q_{2}-q_{1}\right)\left|u_{1}^{(g)}\right|^{2} \mathrm{~d} x \\
& \geq c \int_{B} k^{2}\left|u_{1}^{(g)}\right|^{2} \mathrm{~d} x-C \int_{\Omega \backslash O} k^{2}\left|u_{1}^{(g)}\right|^{2} \mathrm{~d} x
\end{aligned}
$$

for all $g \in V^{\perp}$, where $C:=\left(\left\|q_{1}\right\|_{L^{\infty}(\Omega)}+\left\|q_{2}\right\|_{L^{\infty}(\Omega)}\right)$ and $u_{1}^{(g)}$ solves (23) with $q=q_{1}$.

However, using the localized potentials from Theorem 4.1 with $D:=\bar{\Omega} \backslash O$, there must exist a Neumann datum $g \in V^{\perp}$ with

$$
c \int_{B} k^{2}\left|u_{1}^{(g)}\right|^{2} \mathrm{~d} x>C \int_{\Omega \backslash O} k^{2}\left|u_{1}^{(g)}\right|^{2} \mathrm{~d} x,
$$

which contradicts the above inequality. Hence, $\Lambda\left(q_{2}\right)-\Lambda\left(q_{1}\right)$ must have infinitely many negative eigenvalues, and in particular $\Lambda\left(q_{2}\right) \neq \Lambda\left(q_{1}\right)$.

Proof of Theorem 1.1. The result is an immediate consequence of Theorem 5.1.

Theorem 5.1 shows that two scattering coefficient functions can be distinguished from knowledge of the partial boundary measurements if their difference is of definite sign in a neighborhood of $\Sigma$ (or any open subset of $\Sigma$ since $\Lambda(\Sigma)$ determines the boundary measurements on all smaller parts). This definite sign condition is satisfied for piecewise-analytic functions, see, e.g., [Harrach and Ullrich 2013, Theorem A.1], but the authors are not aware of other named function spaces, with less regularity, where infinite oscillations between positive and negative values when approaching the boundary can be ruled out. In the following corollary the term piecewise-analytic is understood with respect to a partition in finitely many subdomains with piecewise $C^{\infty}$-boundaries; see [Harrach and Ullrich 2013] for a precise definition. 
Corollary 5.2. If $q_{1}-q_{2}$ is piecewise-analytic on $\Omega$ then

$$
\Lambda\left(q_{1}\right)=\Lambda\left(q_{2}\right) \quad \text { if and only if } \quad q_{1}=q_{2} .
$$

Proof. This follows from Theorem 1.1 and [Harrach and Ullrich 2013, Theorem A.1].

\section{Detecting the support of a scatterer}

We will now show that an unknown scatterer, where the refraction index is either higher or lower than an otherwise homogeneous background value, can be reconstructed by simple monotonicity comparisons.

6A. Scatterer detection by monotonicity tests. As before, let $\Omega \subset \mathbb{R}^{n}, n \geq 2$, be a bounded domain with Lipschitz boundary. The domain is assumed to contain an open set (the scatterer) $D \subseteq \Omega$ with $\bar{D} \subset \Omega$ and connected complement $\Omega \backslash \bar{D}$. We assume that the scattering index satisfies $q(x)=1$ in $\Omega \backslash D$ (a.e.) and that there exist constants $q_{\min }, q_{\max } \in \mathbb{R}$ so that either

$$
1<q_{\min } \leq q(x) \leq q_{\max } \text { for all } x \in D \text { (a.e.) }
$$

or

$$
q_{\min } \leq q(x) \leq q_{\max }<1 \quad \text { for all } x \in D \text { (a.e.). }
$$

$\Lambda(q)$ denotes the Neumann-to-Dirichlet operator for the domain containing the scatterer, and $\Lambda(1)$ is the Neumann-to-Dirichlet operator for a homogeneous domain with $q \equiv 1$. For both cases, we assume that $k>0$ is not a resonance.

For an open set $B \subseteq \Omega$ (e.g., a small ball), we define the operator

$$
T_{B}: L^{2}(\Sigma) \rightarrow L^{2}(\Sigma), \quad \int_{\Sigma} g T_{B} h \mathrm{~d} s:=\int_{B} k^{2} u_{1}^{(g)} u_{1}^{(h)} \mathrm{d} x,
$$

where $u_{1}^{(g)}, u_{1}^{(h)} \in H^{1}(\Omega)$ solve (2) with $q \equiv 1$ and Neumann boundary data $g$ and $h$ respectively. Obviously, $T_{B}$ is a compact self-adjoint linear operator.

The following two theorems show that $D$ can be reconstructed by comparing $\Lambda(q)-\Lambda(1)$ with $T_{B}$ in the sense of the Loewner order up to finitely many eigenvalues introduced in Section 3A.

Theorem 6.1. Let

$$
1<q_{\min } \leq q(x) \leq q_{\max } \quad \text { for all } x \in D(\text { a.e. }),
$$

and let $d\left(q_{\max }\right)$ be defined as in Lemma 2.1 (which also equals the number of Neumann eigenvalues of the Laplacian $\Delta$ that are larger than $-k^{2} q_{\max }$; see Corollary 3.11).

(a) If $B \subseteq D$ then

$$
\alpha T_{B} \leq_{d\left(q_{\max }\right)} \Lambda(q)-\Lambda(1) \quad \text { for all } \alpha \leq q_{\min }-1 .
$$

(b) If $B \nsubseteq D$ then, for all $\alpha>0, \Lambda(q)-\Lambda(1)-\alpha T_{B}$ has infinitely many negative eigenvalues.

Theorem 6.2. Let

$$
q_{\min } \leq q(x) \leq q_{\max }<1 \quad \text { for all } x \in D(\text { a.e. }),
$$


and let $d(1)$ be defined as in Lemma 2.1 (which also equals the number of Neumann eigenvalues of the Laplacian $\Delta$ that are larger than $-k^{2}$; see Corollary 3.11$)$.

(a) If $B \subseteq D$ then there exists $\alpha_{\max }>0$ such that

$$
\alpha T_{B} \leq_{d(1)} \Lambda(1)-\Lambda(q) \text { for all } \alpha \leq \alpha_{\max }
$$

(b) If $B \nsubseteq D$ then, for all $\alpha>0, \Lambda(1)-\Lambda(q)-\alpha T_{B}$ has infinitely many negative eigenvalues.

6B. Proof of Theorems 6.1 and 6.2. We prove both results by combining the monotonicity relations and localized potentials results from the last subsections.

Proof of Theorem 6.1. By the monotonicity relation in Theorem 3.5 there exists a subspace $V \subset L^{2}(\Sigma)$ with $\operatorname{dim}(V) \leq d(q) \leq d\left(q_{\max }\right)$ (see Corollary 3.11) and

$$
\int_{\Sigma} g(\Lambda(q)-\Lambda(1)) g \mathrm{~d} s \geq \int_{\Omega} k^{2}(q-1)\left|u_{1}^{(g)}\right|^{2} \mathrm{~d} x \quad \text { for all } g \in V^{\perp} .
$$

If $B \subseteq D$ and $\alpha \leq q_{\min }-1$, then $q-1 \geq \alpha \chi_{B}$, so that for all $g \in L^{2}(\Sigma)$

$$
\int_{\Omega} k^{2}(q-1)\left|u_{1}^{(g)}\right|^{2} \mathrm{~d} x \geq \int_{B} k^{2} \alpha\left|u_{1}^{(g)}\right|^{2} \mathrm{~d} x=\alpha \int_{\Sigma} g T_{B} g \mathrm{~d} s .
$$

Hence, if $B \subseteq D$ and $\alpha \leq q_{\min }-1$, then

which proves (a).

$$
\int_{\Sigma} g(\Lambda(q)-\Lambda(1)) g \mathrm{~d} s \geq \alpha \int_{\Sigma} g T_{B} g \mathrm{~d} s \quad \text { for all } g \in V^{\perp}
$$

To prove (b) by contradiction, let $B \nsubseteq D, \alpha>0$, and assume that

$$
\Lambda(q)-\Lambda(1) \geq_{\text {fin }} \alpha T_{B}
$$

Using the monotonicity relation in Remark 3.6 together with Theorem 4.2, there exists a finite-dimensional subspace $V \subset L^{2}(\Sigma)$ and a constant $C>0$, so that for all $g \in V^{\perp}$

$$
\int_{\Sigma} g(\Lambda(q)-\Lambda(1)) g \mathrm{~d} s \leq \int_{D} k^{2}(q-1)\left|u_{q}^{(g)}\right|^{2} \mathrm{~d} x \leq C \int_{D} k^{2}(q-1)\left|u_{1}^{(g)}\right|^{2} \mathrm{~d} x .
$$

Combining (24) and (25) using the transitivity result from Lemma 3.4, there exists a finite-dimensional subspace $\tilde{V} \subset L^{2}(\Sigma)$ with

$$
\alpha \int_{B} k^{2}\left|u_{1}^{(g)}\right|^{2} \mathrm{~d} x \leq C \int_{D} k^{2}(q-1)\left|u_{1}^{(g)}\right|^{2} \mathrm{~d} x \quad \text { for all } g \in \widetilde{V}^{\perp} .
$$

However, this is contradicted by the localized potentials result in Theorem 4.1, which guarantees the existence of a sequence $\left(g_{j}\right)_{j \in \mathbb{N}} \subset \widetilde{V}^{\perp}$ with

$$
\int_{B}\left|u_{1}^{\left(g_{j}\right)}\right|^{2} \mathrm{~d} x \rightarrow \infty \text { and } \quad \int_{D}\left|u_{1}^{\left(g_{j}\right)}\right|^{2} \mathrm{~d} x \rightarrow 0 .
$$

Hence, $\Lambda(q)-\Lambda(1)-\alpha T_{B}$ cannot have only finitely many negative eigenvalues. 
Proof of Theorem 6.2. The proof is analogous to that of Theorem 6.1. We state it for the sake of completeness. Let

$$
q_{\min } \leq q(x) \leq q_{\max }<1 \text { for all } x \in D \text { (a.e.) }
$$

If $B \subseteq D$, then by the monotonicity relation in Remark 3.6, together with Theorem 4.2, we have

$$
\begin{aligned}
\int_{\Sigma} g(\Lambda(q)-\Lambda(1)) g \mathrm{~d} s & \leq d(1) \int_{\Omega} k^{2}(q-1)\left|u_{q}^{(g)}\right|^{2} \mathrm{~d} x \leq-\int_{D} k^{2}\left(1-q_{\max }\right)\left|u_{q}^{(g)}\right|^{2} \mathrm{~d} x \\
& \leq-c\left(1-q_{\max }\right) \int_{D} k^{2}\left|u_{1}^{(g)}\right|^{2} \mathrm{~d} x \leq-c\left(1-q_{\max }\right) \int_{B} k^{2}\left|u_{1}^{(g)}\right|^{2} \mathrm{~d} x \\
& =-c\left(1-q_{\max }\right) \int_{\Sigma} g T_{B} g \mathrm{~d} s,
\end{aligned}
$$

with a constant $c>0$ from Theorem 4.2. This shows that $B \subseteq D$ implies

$$
\alpha T_{B} \leq_{d(1)} \Lambda(1)-\Lambda(q) \text { for all } \alpha \leq c\left(1-q_{\max }\right)=: \alpha_{\max },
$$

so that (a) is proven.

To prove (b) by contradiction, let $B \nsubseteq D, \alpha>0$, and assume that

$$
\Lambda(1)-\Lambda(q) \geq_{\text {fin }} \alpha T_{B} .
$$

By the monotonicity relation in Theorem 3.5, we have

$$
\int_{\Sigma} g(\Lambda(1)-\Lambda(q)) g \mathrm{~d} s \leq_{\text {fin }} \int_{D} k^{2}(1-q)\left|u_{1}^{(g)}\right|^{2} \mathrm{~d} x .
$$

Combining (26) and (27) using the transitivity result from Lemma 3.4, we have

$$
\alpha \int_{B} k^{2}\left|u_{1}^{(g)}\right|^{2} \mathrm{~d} x \leq_{\text {fin }} \int_{D} k^{2}(1-q)\left|u_{1}^{(g)}\right|^{2} \mathrm{~d} x .
$$

However, this is contradicted by Theorem 4.1, which guarantees (for each finite-dimensional space $\left.V \subset L^{2}(\Sigma)\right)$ the existence of a sequence $\left(g_{j}\right)_{j \in \mathbb{N}} \subset V^{\perp}$ with

$$
\int_{B}\left|u_{0}^{\left(g_{j}\right)}\right|^{2} \mathrm{~d} x \rightarrow \infty \text { and } \int_{D}\left|u_{0}^{\left(g_{j}\right)}\right|^{2} \mathrm{~d} x \rightarrow 0 .
$$

Hence, $\Lambda(1)-\Lambda(q)-\alpha T_{B}$ cannot have only finitely many negative eigenvalues, which shows (b).

6C. Remarks and extensions. We finish this section with some remarks on possible extensions of our results. Theorems 6.1 and 6.2 hold with analogous proofs also for the case that the homogeneous background scattering index is replaced by a known inhomogeneous function $q_{0} \in L^{\infty}(\Omega)$. Using the concept of the inner and outer support from [Harrach and Ullrich 2013] (see also [Kusiak and Sylvester 2003; Gebauer and Hyvönen 2008; Harrach and Seo 2010] for the origins of this concept), we can also treat the case where $\Omega \backslash \bar{D}$ is not connected or where there is no clear jump of the scattering index. The monotonicity tests will then determine $D$ up to the difference of the inner and outer support. Moreover, the so-called indefinite case that the domain contains scatterers with higher and lower refractive indices can be treated by shrinking a large test region analogously to [Harrach and Ullrich 2013]; see also [Garde and Staboulis 2019]. 


\section{Acknowledgements}

Pohjola and Salo were supported by the Academy of Finland (Finnish Centre of Excellence in Inverse Problems Research, grant number 284715) and by an ERC Starting Grant (grant number 307023).

\section{References}

[Alessandrini 1990] G. Alessandrini, "Singular solutions of elliptic equations and the determination of conductivity by boundary measurements", J. Differential Equations 84:2 (1990), 252-272. MR Zbl

[Alessandrini 2012] G. Alessandrini, "Strong unique continuation for general elliptic equations in 2D", J. Math. Anal. Appl. 386:2 (2012), 669-676. MR Zbl

[Ammari and Uhlmann 2004] H. Ammari and G. Uhlmann, "Reconstruction of the potential from partial Cauchy data for the Schrödinger equation", Indiana Univ. Math. J. 53:1 (2004), 169-183. MR Zbl

[Arnold and Harrach 2013] L. Arnold and B. Harrach, "Unique shape detection in transient eddy current problems", Inverse Problems 29:9 (2013), art. id. 095004. MR Zbl

[Astala and Päivärinta 2006] K. Astala and L. Päivärinta, "Calderón's inverse conductivity problem in the plane", Ann. of Math. (2) 163:1 (2006), 265-299. MR Zbl

[Astala et al. 2009] K. Astala, T. Iwaniec, and G. Martin, Elliptic partial differential equations and quasiconformal mappings in the plane, Princeton Math. Series 48, Princeton Univ. Press, 2009. MR Zbl

[Barth et al. 2017] A. Barth, B. Harrach, N. Hyvönen, and L. Mustonen, "Detecting stochastic inclusions in electrical impedance tomography", Inverse Problems 33:11 (2017), art. id. 115012. MR Zbl

[Brander et al. 2018] T. Brander, B. Harrach, M. Kar, and M. Salo, "Monotonicity and enclosure methods for the $p$-Laplace equation", SIAM J. Appl. Math. 78:2 (2018), 742-758. MR Zbl

[Brühl 2001] M. Brühl, "Explicit characterization of inclusions in electrical impedance tomography", SIAM J. Math. Anal. 32:6 (2001), 1327-1341. MR Zbl

[Brühl and Hanke 2000] M. Brühl and M. Hanke, "Numerical implementation of two noniterative methods for locating inclusions by impedance tomography", Inverse Problems 16:4 (2000), 1029-1042. MR Zbl

[Calderón 1980] A. P. Calderón, "On an inverse boundary value problem", pp. 65-73 in Seminar on numerical analysis and its applications to continuum physics (Rio de Janeiro, 1980), edited by W. H. Meyer and M. A. Raupp, Coleção Atas 12, Soc. Brasil. Mat., Rio de Janeiro, 1980. MR

[Calderón 2006] A. P. Calderón, “On an inverse boundary value problem”, Comput. Appl. Math. 25:2-3 (2006), 133-138. MR $\mathrm{Zbl}$

[Caro and Rogers 2016] P. Caro and K. M. Rogers, "Global uniqueness for the Calderón problem with Lipschitz conductivities", Forum Math. Pi 4 (2016), art. id. e2. MR Zbl

[Druskin 1998] V. Druskin, "On the uniqueness of inverse problems from incomplete boundary data", SIAM J. Appl. Math. 58:5 (1998), 1591-1603. MR Zbl

[de Figueiredo and Gossez 1992] D. G. de Figueiredo and J.-P. Gossez, "Strict monotonicity of eigenvalues and unique continuation”, Comm. Partial Differential Equations 17:1-2 (1992), 339-346. MR Zbl

[Garde 2018] H. Garde, "Comparison of linear and non-linear monotonicity-based shape reconstruction using exact matrix characterizations", Inverse Probl. Sci. Eng. 26:1 (2018), 33-50. MR Zbl

[Garde and Staboulis 2017] H. Garde and S. Staboulis, "Convergence and regularization for monotonicity-based shape reconstruction in electrical impedance tomography", Numer. Math. 135:4 (2017), 1221-1251. MR Zbl

[Garde and Staboulis 2019] H. Garde and S. Staboulis, "The regularized monotonicity method: detecting irregular indefinite inclusions", Inverse Probl. Imaging 13:1 (2019), 93-116. Zbl

[Gebauer 2008] B. Gebauer, "Localized potentials in electrical impedance tomography”, Inverse Probl. Imaging 2:2 (2008), 251-269. MR Zbl 
[Gebauer and Hyvönen 2008] B. Gebauer and N. Hyvönen, "Factorization method and inclusions of mixed type in an inverse elliptic boundary value problem", Inverse Probl. Imaging 2:3 (2008), 355-372. MR Zbl

[Griesmaier and Harrach 2018] R. Griesmaier and B. Harrach, "Monotonicity in inverse medium scattering on unbounded domains", SIAM J. Appl. Math. 78:5 (2018), 2533-2557. MR Zbl

[Guillarmou and Tzou 2013] C. Guillarmou and L. Tzou, "The Calderón inverse problem in two dimensions", pp. 119-166 in Inverse problems and applications: inside out, II, edited by G. Uhlmann, Math. Sci. Res. Inst. Publ. 60, Cambridge Univ. Press, 2013. MR Zbl

[Haberman and Tataru 2013] B. Haberman and D. Tataru, "Uniqueness in Calderón's problem with Lipschitz conductivities", Duke Math. J. 162:3 (2013), 497-516. MR Zbl

[Hadi and Tsouli 2001] I. E. Hadi and N. Tsouli, "Strong unique continuation of eigenfunctions for $p$-Laplacian operator", Int. J. Math. Math. Sci. 25:3 (2001), 213-216. MR Zbl

[Harrach 2009] B. Harrach, "On uniqueness in diffuse optical tomography”, Inverse Problems 25:5 (2009), art. id. 055010. MR $\mathrm{Zbl}$

[Harrach 2012] B. Harrach, "Simultaneous determination of the diffusion and absorption coefficient from boundary data", Inverse Probl. Imaging 6:4 (2012), 663-679. MR Zbl

[Harrach 2013] B. Harrach, "Recent progress on the factorization method for electrical impedance tomography", Comput. Math. Methods Med. 2013 (2013), art. id. 425184. MR Zbl

[Harrach 2019] B. Harrach, "Uniqueness and Lipschitz stability in electrical impedance tomography with finitely many electrodes", Inverse Problems 35:2 (2019), art. id. 024005. Zbl

[Harrach and Lin 2017] B. Harrach and Y.-H. Lin, "Monotonicity-based inversion of the fractional Schrödinger equation, I: Positive potentials", preprint, 2017. To appear in SIAM J. Math. Anal. arXiv

[Harrach and Lin 2019] B. Harrach and Y.-H. Lin, "Monotonicity-based inversion of the fractional Schrödinger equation, II: General potentials and stability", preprint, 2019. arXiv

[Harrach and Meftahi 2019] B. Harrach and H. Meftahi, "Global uniqueness and Lipschitz-stability for the inverse Robin transmission problem”, SIAM J. Appl. Math. 79:2 (2019), 525-550. MR Zbl

[Harrach and Minh 2016] B. Harrach and M. N. Minh, "Enhancing residual-based techniques with shape reconstruction features in electrical impedance tomography", Inverse Problems 32:12 (2016), art. id. 125002. MR Zbl

[Harrach and Minh 2018] B. Harrach and M. N. Minh, "Monotonicity-based regularization for phantom experiment data in electrical impedance tomography", pp. 107-120 in New trends in parameter identification for mathematical models (Rio de Janeiro, 2017), edited by B. Hofmann et al., Birkhäuser, Cham, 2018. MR Zbl

[Harrach and Seo 2010] B. Harrach and J. K. Seo, "Exact shape-reconstruction by one-step linearization in electrical impedance tomography", SIAM J. Math. Anal. 42:4 (2010), 1505-1518. MR Zbl

[Harrach and Ullrich 2013] B. Harrach and M. Ullrich, "Monotonicity-based shape reconstruction in electrical impedance tomography”, SIAM J. Math. Anal. 45:6 (2013), 3382-3403. MR Zbl

[Harrach and Ullrich 2015] B. Harrach and M. Ullrich, "Resolution guarantees in electrical impedance tomography", IEEE Trans. Med. Imaging 34:7 (2015), 1513-1521. Zbl

[Harrach and Ullrich 2017] B. Harrach and M. Ullrich, "Local uniqueness for an inverse boundary value problem with partial data", Proc. Amer. Math. Soc. 145:3 (2017), 1087-1095. MR Zbl

[Harrach et al. 2015] B. Harrach, E. Lee, and M. Ullrich, "Combining frequency-difference and ultrasound modulated electrical impedance tomography”, Inverse Problems 31:9 (2015), art. id. 095003. MR Zbl

[Harrach et al. 2017] B. Harrach, V. Pohjola, and M. Salo, "The monotonicity method for inverse scattering", Oberwolfach Rep. 24 (2017), 57-60.

[Harrach et al. 2018] B. Harrach, Y.-H. Lin, and H. Liu, "On localizing and concentrating electromagnetic fields", SIAM J. Appl. Math. 78:5 (2018), 2558-2574. MR Zbl

[Harrach et al. 2019] B. Harrach, V. Pohjola, and M. Salo, "Dimension bounds in monotonicity methods for the Helmholtz equation", preprint, 2019. To appear in SIAM J. Math. Anal. arXiv 
[Hörmander 1985] L. Hörmander, The analysis of linear partial differential operators, III: Pseudodifferential operators, Grundlehren der Math. Wissenschaften 274, Springer, 1985. MR Zbl

[Ikehata 1998] M. Ikehata, "Size estimation of inclusion”, J. Inverse Ill-Posed Probl. 6:2 (1998), 127-140. MR Zbl

[Ikehata 1999] M. Ikehata, "Identification of the shape of the inclusion having essentially bounded conductivity", J. Inverse Ill-Posed Probl. 7:6 (1999), 533-540. MR Zbl

[Imanuvilov et al. 2010] O. Y. Imanuvilov, G. Uhlmann, and M. Yamamoto, "The Calderón problem with partial data in two dimensions", J. Amer. Math. Soc. 23:3 (2010), 655-691. MR Zbl

[Imanuvilov et al. 2015] O. Y. Imanuvilov, G. Uhlmann, and M. Yamamoto, "The Neumann-to-Dirichlet map in two dimensions", Adv. Math. 281 (2015), 578-593. MR Zbl

[Isakov 1988] V. Isakov, "On uniqueness of recovery of a discontinuous conductivity coefficient", Comm. Pure Appl. Math. 41:7 (1988), 865-877. MR Zbl

[Isakov 2007] V. Isakov, "On uniqueness in the inverse conductivity problem with local data", Inverse Probl. Imaging 1:1 (2007), 95-105. MR Zbl

[Kang et al. 1997] H. Kang, J. K. Seo, and D. Sheen, "The inverse conductivity problem with one measurement: stability and estimation of size", SIAM J. Math. Anal. 28:6 (1997), 1389-1405. MR Zbl

[Kenig and Salo 2013] C. Kenig and M. Salo, "The Calderón problem with partial data on manifolds and applications", Anal. PDE 6:8 (2013), 2003-2048. MR Zbl

[Kenig and Salo 2014] C. Kenig and M. Salo, "Recent progress in the Calderón problem with partial data", pp. 193-222 in Inverse problems and applications (Irvine, CA, 2012/Hangzhou, 2012), edited by P. Stefanov et al., Contemp. Math. 615, Amer. Math. Soc., Providence, RI, 2014. MR Zbl

[Kenig et al. 2007] C. E. Kenig, J. Sjöstrand, and G. Uhlmann, "The Calderón problem with partial data”, Ann. of Math. (2) 165:2 (2007), 567-591. MR Zbl

[Kirsch 1998] A. Kirsch, "Characterization of the shape of a scattering obstacle using the spectral data of the far field operator", Inverse Problems 14:6 (1998), 1489-1512. MR Zbl

[Kirsch and Grinberg 2008] A. Kirsch and N. Grinberg, The factorization method for inverse problems, Oxford Lecture Series Math. Appl. 36, Oxford Univ. Press, 2008. MR Zbl

[Kohn and Vogelius 1984] R. Kohn and M. Vogelius, "Determining conductivity by boundary measurements", Comm. Pure Appl. Math. 37:3 (1984), 289-298. MR Zbl

[Kohn and Vogelius 1985] R. V. Kohn and M. Vogelius, "Determining conductivity by boundary measurements, II: Interior results", Comm. Pure Appl. Math. 38:5 (1985), 643-667. MR Zbl

[Krupchyk and Uhlmann 2016] K. Krupchyk and G. Uhlmann, "The Calderón problem with partial data for conductivities with 3/2 derivatives", Comm. Math. Phys. 348:1 (2016), 185-219. MR Zbl

[Kusiak and Sylvester 2003] S. Kusiak and J. Sylvester, "The scattering support", Comm. Pure Appl. Math. 56:11 (2003), 1525-1548. MR Zbl

[Lax 2002] P. D. Lax, Functional analysis, Wiley, New York, 2002. MR Zbl

[Maffucci et al. 2016] A. Maffucci, A. Vento, S. Ventre, and A. Tamburrino, "A novel technique for evaluating the effective permittivity of inhomogeneous interconnects based on the monotonicity property", IEEE Trans. Components Packaging Manuf. Tech. 6:9 (2016), 1417-1427.

[Nachman 1996] A. I. Nachman, "Global uniqueness for a two-dimensional inverse boundary value problem", Ann. of Math. (2) 143:1 (1996), 71-96. MR Zbl

[Reed and Simon 1972] M. Reed and B. Simon, Methods of modern mathematical physics, I: Functional analysis, Academic Press, New York, 1972. MR Zbl

[Regbaoui 2001] R. Regbaoui, “Unique continuation from sets of positive measure”, pp. 179-190 in Carleman estimates and applications to uniqueness and control theory (Cortona, Italy, 1999), edited by F. Colombini and C. Zuily, Progr. Nonlinear Differential Equations Appl. 46, Birkhäuser, Boston, 2001. MR Zbl 
[Su et al. 2017] Z. Su, L. Udpa, G. Giovinco, S. Ventre, and A. Tamburrino, "Monotonicity principle in pulsed eddy current testing and its application to defect sizing", pp. art. id. 48-02 in Int. Applied Computational Electromagnetics Society Symposium (Florence, 2017), IEEE, Piscataway, NJ, 2017.

[Sylvester and Uhlmann 1987] J. Sylvester and G. Uhlmann, "A global uniqueness theorem for an inverse boundary value problem", Ann. of Math. (2) 125:1 (1987), 153-169. MR Zbl

[Tamburrino and Rubinacci 2002] A. Tamburrino and G. Rubinacci, "A new non-iterative inversion method for electrical resistance tomography", Inverse Problems 18:6 (2002), 1809-1829. MR Zbl

[Tamburrino et al. 2016] A. Tamburrino, Z. Su, S. Ventre, L. Udpa, and S. S. Udpa, "Monotonicity based imaging method in time domain eddy current testing", pp. 1-8 in Electromagnetic nondestructive evaluation, XIX (Sendai, Japan, 2015), edited by N. Yusa et al., Studies Appl. Electromag. Mech. 41, IOS Press, Amsterdam, 2016.

[Ventre et al. 2017] S. Ventre, A. Maffucci, F. Caire, N. Le Lostec, A. Perrotta, G. Rubinacci, B. Sartre, A. Vento, and A. Tamburrino, "Design of a real-time eddy current tomography system", IEEE Trans. Magnetics 53:3 (2017), art. id. 6200408.

[Zhou et al. 2018] L. Zhou, B. Harrach, and J. K. Seo, "Monotonicity-based electrical impedance tomography for lung imaging", Inverse Problems 34:4 (2018), art. id. 045005. MR Zbl

Received 27 Nov 2017. Revised 20 Jul 2018. Accepted 20 Nov 2018.

BASTIAN HARRACH: harrach@math.uni-frankfurt.de

Institute of Mathematics, Goethe University Frankfurt, Frankfurt, Germany

VALTER POHJOLA: valter.pohjola@gmail.com

Research Unit of Mathematical Sciences, University of Oulu, Oulu, Finland

MiKKo SALO: mikko.j.salo@jyu.fi

Department of Mathematics and Statistics, University of Jyväskylä, Jyväskylä, Finland 


\title{
Analysis \& PDE
}

msp.org/apde

\section{EDITORS}

\author{
EDITOR-IN-CHIEF \\ Patrick Gérard \\ patrick.gerard@math.u-psud.fr \\ Université Paris Sud XI \\ Orsay, France
}

BOARD OF EDITORS

\begin{tabular}{|c|c|}
\hline Massimiliano Berti & $\begin{array}{l}\text { Scuola Intern. Sup. di Studi Avanzati, Italy } \\
\text { berti@ sissa.it }\end{array}$ \\
\hline Michael Christ & $\begin{array}{l}\text { University of California, Berkeley, USA } \\
\text { mchrist@ math.berkeley.edu }\end{array}$ \\
\hline Charles Fefferman & $\begin{array}{l}\text { Princeton University, USA } \\
\text { cf@math.princeton.edu }\end{array}$ \\
\hline Ursula Hamenstaedt & $\begin{array}{l}\text { Universität Bonn, Germany } \\
\text { ursula@math.uni-bonn.de }\end{array}$ \\
\hline Vadim Kaloshin & $\begin{array}{l}\text { University of Maryland, USA } \\
\text { vadim.kaloshin@gmail.com }\end{array}$ \\
\hline Herbert Koch & $\begin{array}{l}\text { Universität Bonn, Germany } \\
\text { koch@math.uni-bonn.de }\end{array}$ \\
\hline Izabella Laba & $\begin{array}{l}\text { University of British Columbia, Canada } \\
\text { ilaba@math.ubc.ca }\end{array}$ \\
\hline Richard B. Melrose & $\begin{array}{l}\text { Massachussets Inst. of Tech., USA } \\
\text { rbm@math.mit.edu }\end{array}$ \\
\hline Frank Merle & $\begin{array}{l}\text { Université de Cergy-Pontoise, France } \\
\text { Frank.Merle@u-cergy.fr }\end{array}$ \\
\hline William Minicozzi II & $\begin{array}{l}\text { Johns Hopkins University, USA } \\
\text { minicozz@math.jhu.edu }\end{array}$ \\
\hline Clément Mouhot & $\begin{array}{l}\text { Cambridge University, UK } \\
\text { c.mouhot@dpmms.cam.ac.uk }\end{array}$ \\
\hline Werner Müller & $\begin{array}{l}\text { Universität Bonn, Germany } \\
\text { mueller@math.uni-bonn.de }\end{array}$ \\
\hline
\end{tabular}

Gilles Pisier Texas A\&M University, and Paris 6 pisier@math.tamu.edu

Tristan Rivière ETH, Switzerland riviere@math.ethz.ch

Igor Rodnianski Princeton University, USA irod@math.princeton.edu

Sylvia Serfaty New York University, USA serfaty@cims.nyu.edu

Yum-Tong Siu Harvard University, USA siu@math.harvard.edu

Terence Tao University of California, Los Angeles, USA tao@math.ucla.edu

Michael E. Taylor Univ. of North Carolina, Chapel Hill, USA met@math.unc.edu

Gunther Uhlmann University of Washington, USA gunther@math.washington.edu

András Vasy Stanford University, USA andras@math.stanford.edu

Dan Virgil Voiculescu University of California, Berkeley, USA dvv@math.berkeley.edu

Steven Zelditch Northwestern University, USA zelditch@math.northwestern.edu

Maciej Zworski University of California, Berkeley, USA zworski@math.berkeley.edu

PRODUCTION

production@msp.org

Silvio Levy, Scientific Editor

See inside back cover or msp.org/apde for submission instructions.

The subscription price for 2019 is US $\$ 310 /$ year for the electronic version, and $\$ 520 /$ year $(+\$ 60$, if shipping outside the US) for print and electronic. Subscriptions, requests for back issues from the last three years and changes of subscriber address should be sent to MSP.

Analysis \& PDE (ISSN 1948-206X electronic, 2157-5045 printed) at Mathematical Sciences Publishers, 798 Evans Hall \#3840, c/o University of California, Berkeley, CA 94720-3840, is published continuously online. Periodical rate postage paid at Berkeley, CA 94704, and additional mailing offices.

APDE peer review and production are managed by EditFlow ${ }^{\circledR}$ from MSP.

\section{PUBLISHED BY}

\section{mathematical sciences publishers}

nonprofit scientific publishing

http://msp.org/

(C) 2019 Mathematical Sciences Publishers 


\section{ANALYSIS \& PDE}

\section{Volume 12 No. $7 \quad 2019$}

Generalized $q$-gaussian von Neumann algebras with coefficients, I: Relative strong solidity 1643 MARIUS JUNGE and BOGDAN UDREA

Complex interpolation and Calderón-Mityagin couples of Morrey spaces

MiECZYSŁAW MASTYŁo and YoshIHIRO SAWANO

Monotonicity and local uniqueness for the Helmholtz equation

Bastian Harrach, Valter Pohjola and Mikko Salo

Solutions of the 4-species quadratic reaction-diffusion system are bounded and $C^{\infty}$-smooth, 1773 in any space dimension

M. Cristina Caputo, Thierry Goudon and Alexis F. Vasseur

Spacelike radial graphs of prescribed mean curvature in the Lorentz-Minkowski space

DENIS BONHEURE and ALESSANDRO IACOPETTI

Square function estimates, the BMO Dirichlet problem, and absolute continuity of harmonic 1843 measure on lower-dimensional sets

SVITLANA MAYBORODA and ZIHUi ZHAO 